\title{
In-plane crashworthiness of re-entrant hierarchical honeycombs
}

\section{with negative Poisson's ratio}

\author{
Hailun. Tan ${ }^{\text {a }}$, Z. C. He ${ }^{\mathrm{a}^{*}}$, K.X. $\mathrm{Li}^{\mathrm{a}}$, Eric Li ${ }^{\mathrm{b}}$, A. G. Cheng ${ }^{\mathrm{a}}$, Bing Xu ${ }^{\mathrm{c}}$ \\ a State Key Laboratory of Advanced Design and Manufacturing for Vehicle Body, Hunan University, \\ Changsha, 410082 P. R. China \\ ${ }^{\mathrm{b}}$ School of Science, Engineering \& Design, Teesside University, Middleborough, United Kingdom \\ ${ }^{\mathrm{c}}$ Technology Development Center, SAIC GM Wuling Automobile Co., Ltd., Liuzhou, People's Republic \\ of China
}

\begin{abstract}
Both auxetic structures and hierarchical honeycombs are marked with lightweight and excellent mechanical properties. Here, we combine the characteristics of auxetic structures and hierarchical honeycombs, and propose two re-entrant hierarchical honeycombs constructed by replacing the cell walls of re-entrant honeycombs with regular hexagon substructure (RHH) and equilateral triangle substructure (RHT). The honeycombs are subjected to in-plane impact in order to investigate the crashworthiness by using the commercial software LS-DYNA. The plateau stress of RHH and RHT in $x$ and $y$ directions are derived by a two-scale method. The results from numerical simulation indicate that the specific energy absorption of RHT and RHH is improved by up to $292 \%$ and $105 \%$. RHT and RHH improve the mean crushing force value by $298 \%, 108 \%$ respectively compared with the classic re-entrant honeycomb (RH) under quasi-static loading at stress plateau region. The RHT and RHH still have the characteristic of negative Poisson's ratio. Additionally, the parametric studies are further carried out to investigate the effects of impact velocities and relative densities on crashworthiness. All the findings of this study indicate that the proposed two hierarchical honeycombs exhibit an improved crushing performance, and RHT provides the highest energy absorption capacity among all specimens.
\end{abstract}

Key words: hierarchical honeycombs, negative Poisson's ratio, energy absorption capacity

\footnotetext{
* Corresponding author. Tel./fax: +86 18817119011.

E-mail address: hezhicheng815@163.com
}

\section{Introduction}

Honeycomb structures have attracted a lot of attentions due to their excellent mechanical performance [1] and superior heat dissipation capabilities [2]. Honeycombs are extensively used in the field of transportation, aerospace and 
constructions. A lot of works had been put into effect to investigate the in-plane crushing properties and out-of-plane crushing properties of honeycombs. Wierzbicki et al. [3] investigated the out-of-plane crashworthiness of hexagonal honeycomb structure by using super folding element theory. Hu et al. [4] analyzed the in-plane crashworthiness of hexagonal honeycombs based on the cells' collapse, and found that the honeycombs crushing strength increases with the impact velocity. Cricrì et al. [5] studied the behavior of regular hexagonal honeycombs imposed by the in-plane loading, and used the experimental tests and numerical evaluation to validate the results. Papka et al. [6] performed the quasi-static in-plane crashworthiness of hexagonal aluminum honeycombs. Ruan et al. [7] studied the in-plane dynamic behavior of hexagonal aluminum honeycombs by using ABAQUS, and investigated the influence of cell wall thickness and impact velocity. Wu and Jiang [8] carried out a series of experiments to investigate the crushing strength of six types of aluminum honeycombs under out-of-plane loading. Mukhopadhyay et al. [9] developed a mechanics-based concept to probe the frequency-dependence in in-plane elastic moduli of lattice materials. Lin et al. [10] presented a family of new origami crash boxes to improve the ability of energy absorption. Yang et al. [11] developed a series of open-section beams employing origami geometries to provide higher energy absorption.

In recent years, there has been an increasing amount of studies on auxetic re-entrant honeycomb structures. Because of the negative Poisson's ratio, the auxetic structures have distinctive mechanical properties and good energy absorption ability [12]. Liu et al. [13] compared the energy absorption abilities of the re-entrant auxetic honeycomb and the hexagonal honeycomb, and found that the re-entrant honeycomb can absorb more energy when subjected to the same magnitude of crushing strain. 
Zhang et al. [14] discussed the influences of different cell wall aspect ratios and cell-wall angles of auxetic re-entrant honeycombs, and confirmed that plateau stresses are related to impact velocities. Jin et al. [15] systematically analyzed the dynamic properties and blast resistance of sandwich structure with auxetic re-entrant honeycomb cores under blast loading by using the LS-DYNA. Qi et al. [16] found that a combination of the steel plate and an auxetic sandwich panel has a higher specific energy absorption than the conventional honeycomb panels with the same size. Lu et al. [17] found that adding a rib in the unit cell of re-entrant honeycomb configuration can enhance Young's modulus compared with the classical re-entrant honeycomb. Fu et al. [18] investigated a novel auxetic honeycomb with theoretical analysis and numerical simulations, and concluded that both the in-plane Young's modulus and buckling strength are greatly improved compared to the normal re-entrant hexagonal honeycomb. Li et al. [19] proposed two new models by adding two sinusoidal-shaped ribs and vertical ribs into the classical re-entrant honeycomb, and found their higher energy absorption capacity compared with the classical re-entrant honeycomb. Ma et al. [20] studied the effects of geometric features and configuration on dynamic properties of the novel type of cellular material. Ingrole et al. [21] conducted a comparative study of five different auxetic structures, and studied the deformation and failure modes of these structures, and found that the new auxetic-strut structure has better mechanical properties than other structures. Wang et al. [22] proposed the re-entrant star-shaped honeycomb, and studied the deformation modes under different impact velocities, and found that the structure exhibits excellent impact resistance with the same cell wall thickness compared with classical re-entrant honeycomb and star-shaped honeycomb. Mukhopadhyay et al. [23, 24] developed an analytical framework to predict the equivalent in-plane elastic moduli of 
irregular auxetic honeycombs and the effect of viscoelasticity on irregular hexagonal lattices.

The study of hierarchical honeycombs was also carried out for many years. Sun et al. [25, 26] analyzed the in-plane stiffness of the anisotropic multifunctional hierarchical honeycomb and multifunctional hierarchical honeycombs with negative Poisson's ratio sub-structures based on Euler beam theory. Zhang et al. [27] constructed self-similar regular hexagonal hierarchical honeycombs, and concluded that hierarchical organization could improve crush strength and crush force efficiency by carrying out a series of numerical analyses. Fang et al. [28] and $\mathrm{Wu}$ et al. [29] investigated the out-of-plane crushing performances of hierarchical honeycombs by the experimental and numerical methods, and derived the theoretical expression of mean force and plateau stress by using the simplified super folding element method. Yin et al. [30] compared three different hierarchical honeycombs, and confirmed that the triangular hierarchical honeycomb has the best crushing performance compared to other two hierarchical honeycombs. Xu et al. [31] used the self-similar hierarchical hexagonal columns to improve crashworthiness performance of vehicles. Qiao and Chen [32] proposed a two-scale method to obtain analytical expressions for collapse stresses of a hierarchical honeycomb.

Although both auxetic structures and hierarchical honeycombs have excellent mechanical properties and energy absorption properties, the systematic studies on combining the design of hierarchical structure with auxetic structure are limited. In this study, we introduce the hierarchy into re-entrant honeycombs, and propose two different hierarchical honeycombs by replacing the cell walls of re-entrant honeycombs with relevant cellular substructure, and the in-plane crashworthiness performance is investigated. Section 2 gives the geometric configuration and the finite 
element modeling of the hierarchical honeycombs. Section 3 exhibits the quasi-static collapse of honeycombs. Section 4 discusses the effects of the impact velocities and the relative densities. Several conclusions are made in Section 5.

\section{Mathematical model of hierarchical honeycomb}

\subsection{Structural description}

As depicted in the introduction, the application of hierarchy in re-entrant honeycombs has the potential to further improve their mechanical performance. In this work, re-entrant hierarchical honeycombs with hexagon substructure (RHH) and triangle substructure (RHT) are presented and their crashworthiness performances are studied systematically. Figure 1 shows the cross-section configurations of these honeycombs. The two types of re-entrant hierarchical honeycombs are constructed by replacing the cell walls of re-entrant honeycombs with relevant cellular substructure (regular hexagon substructure and equilateral triangle substructure). The detailed complex geometric configurations of the cellular substructures are shown in Fig. 2. Figure 2 shows the unit cells of the RH, RHH and RHT. All unit cells have the same basic parameters. The lengths parallel and inclined to the horizontal orientation for all units are $H_{0}$ and $L_{0}$, respectively. The angle between the horizontal line and the oblique line is set to $30^{\circ}$. As shown in Fig. $2, l_{h}$ is the edge length of regular hexagon substructure and $l_{t}$ is the edge length of equilateral triangle substructure, $\sqrt{3} \times N_{h_{1}} \times$ $l_{h}$ and $\sqrt{3} \times N_{h_{2}} \times l_{h}$ are the edge length parallel and inclined to the Horizontal orientation of the unit cell of RHH, respectively. $1+N_{h 1}$ and $1+N_{h 2}$ denote the number of substructures along the edge parallel and inclined to the horizontal orientation of the unit cell, respectively. As shown in Fig. 2 (c), $N_{t 1} \times l_{t}$ and $N_{t_{2}} \times l_{t}$ are the edge length parallel and inclined to the horizontal orientation of the unit cell of RHT, 
respectively. $N_{t 1}$ and $N_{t 2}$ are the number of substructures along the edge parallel and inclined to the Horizontal orientation of the unit cell. $t_{0}, t_{h}$ and $t_{t}$ are the thickness of RH, RHH and RHT, respectively. RH, RHH and RHT have the same out-of-plane width which is marked with $b$.

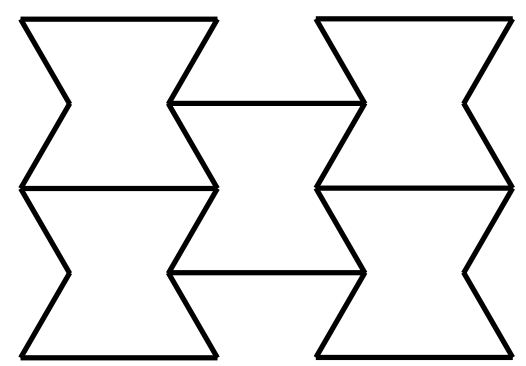

(a)

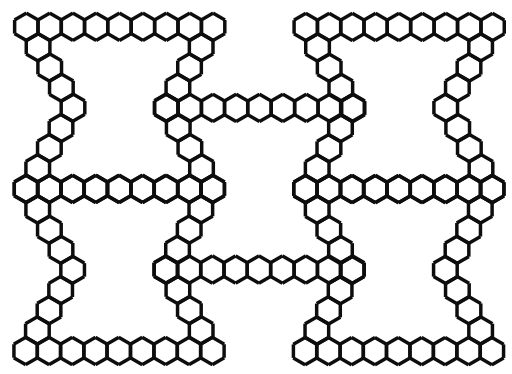

(b)

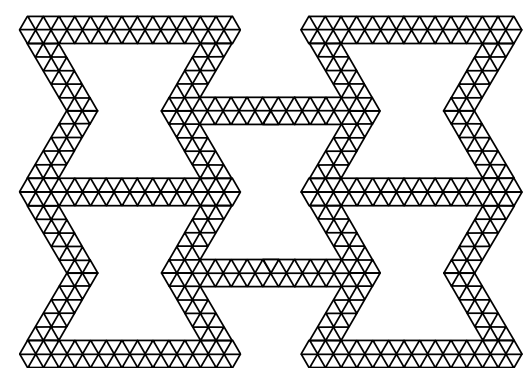

(c)

Fig. 1. Schematic illustrations of re-entrant honeycomb and hierarchical honeycombs. (a) RH. (b)RHH. (c) RHT.

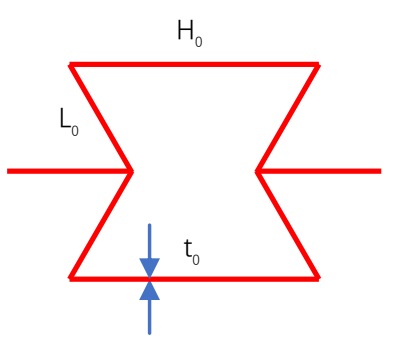

(a)

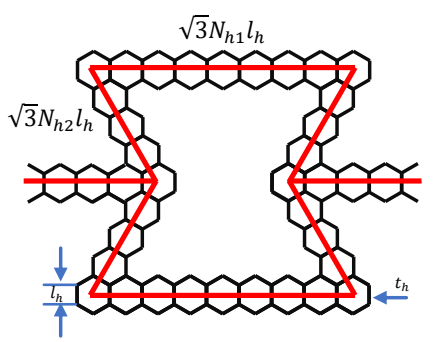

(b)

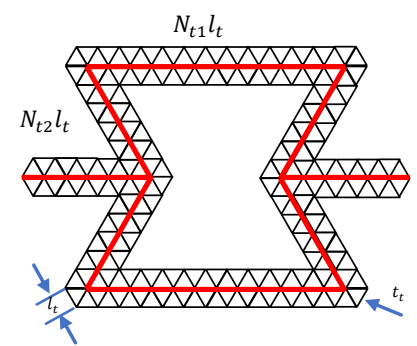

(c)

Fig. 2. The configurations of unit cell of re-entrant honeycomb and hierarchical honeycombs. (a) RH. (b)RHH. (c) RHT.

The relative densities of the RH, RHH, RHT can be calculated as in Eqs. (1), (2) and (3) based on the previous theoretical analysis, respectively.

$$
\begin{aligned}
& \bar{\rho}=\frac{\left(H_{0}+2 L_{0}\right)}{\sqrt{3}\left(H_{0}-L_{0} / 2\right)} \frac{t_{0}}{L_{0}} \\
& \overline{\rho_{h}}=\frac{10\left(N_{h 1}+2 N_{h 2}-2\right)}{3 \sqrt{3} N_{h 2}\left(2 N_{h 1}-N_{h 2}\right)} \frac{t_{h}}{l_{h}} \\
& \overline{\rho_{t}}=\frac{14\left(N_{t 1}+2 N_{t 2}-\frac{20}{7}\right)}{\sqrt{3} N_{t 2}\left(2 N_{t 1}-N_{t 2}\right)} \frac{t_{\mathrm{t}}}{l_{\mathrm{t}}}
\end{aligned}
$$

where $\bar{\rho}, \overline{\rho_{h}}, \overline{\rho_{t}}$ are relative densities of the RH, RHH and RHT, respectively. 


\subsection{Crashworthiness criteria}

It is essential to use crashworthiness indicators to evaluate the capability of energy absorption devices of these structures. Therefore, the energy absorption (EA), the specific energy absorption (SEA) and the mean crushing force (MCF) are employed. EA can be obtained by [33]:

$$
E A(x)=\int_{0}^{l} F(x) d(x)
$$

In Eq. (4), $F(x)$ is the impact force, and the variable $x$ is the crushing displacement. It should be point out that the higher value of EA means higher ability of energy absorption. The SEA is defined that the absorption of energy of per unit mass. It is the most reliable indicator in evaluating the performance of crashworthiness. The SEA is defined as:

$$
S E A(x)=\frac{E A(x)}{m}
$$

where $m$ is the mass of the structure. Usually, the higher value of the MCF is desirable. The MCF is derived from:

$$
\operatorname{MCF}(x)=\frac{E A(x)}{x}
$$

\subsection{Finite element modeling}

Numerical analyses of the in-plane crushing behaviors and energy absorption capacities of the RHH and RHT are systematically simulated by using the nonlinear finite element method LS-DYNA, and the crashworthiness performances of RH are also analyzed for a comparison. The diagrammatic sketches of the numerical models of these honeycombs are illustrated in Fig. 3. In the finite element (FE) models, and 8 $\times 6$ cells are adopted (i.e., 8 cells in the vertical direction, 6 cells in the horizontal direction). All cell walls are meshed by utilizing 2D 4-node shell elements with five 
integration points in thickness. For comparisons, the basic lengths parallel and inclined to the horizontal orientation of the unit cells are set to be the same as $30 \mathrm{~mm}$ and $15 \mathrm{~mm}$. Unless stated otherwise, $N_{h_{1}}=8, N_{h_{2}}=4, N_{t 1}=12, N_{t 2}=6$, $b=10 \mathrm{~mm}$ are adopted in the following FE simulations. The FEM models of RH, RHH and RHT contain 57000 elements, 135120 elements and 428160 elements, respectively. The bottom of the honeycomb is fixed by the rigid plate, and the upper rigid plate compresses the structure with a constant impact speed. The impact speed $V$ varies in the range of 1 to $50 \mathrm{~m} / \mathrm{s}$ in this work in order to study the deformation modes and energy absorption performances of these structures. In all modes, the matrix material of these honeycombs is aluminum alloy AA6063-T6, and the material properties are taken as follows: the density $\rho=2.7 \times 10^{3} \mathrm{~kg} / \mathrm{m}^{3}$, Young's modulus $E=$ 73.0 GPa, tangent modulus $G=28.1 \mathrm{GPa}$, initial yield stress $\sigma_{y}=206 \mathrm{MPa}$, the ultimate stress $\sigma_{u}=257 \mathrm{MPa}$ and Poisson's ratio $v=0.3[34,35]$. Because of the very weak strain rate sensitivity of aluminum alloys, the effect of strain rate is neglected [36].

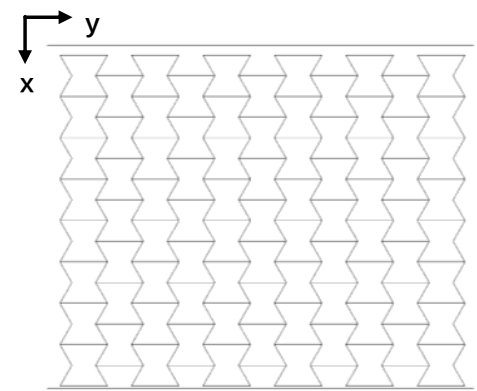

(a)

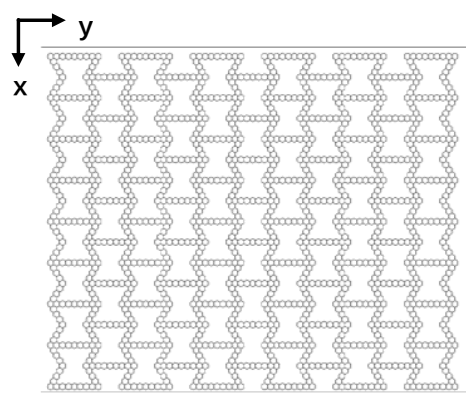

(b)

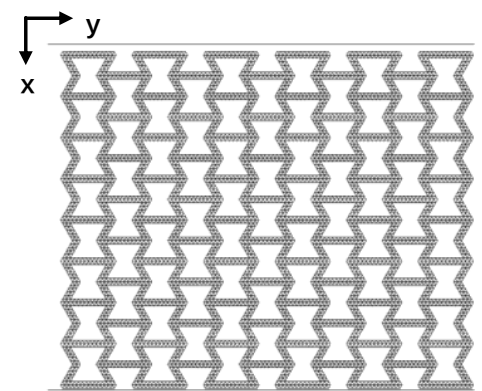

(c)

Fig. 3. Diagrammatic sketches for the analysis models of these honeycombs subject to in-plane loadings (a) RH, (b)RHH, (c)RHT.

There are two types of contact algorithm are used in these models. The first one is an automatic single-surface contact which is used to avoid penetration during deformation, and the other one is an automatic surface-to-surface contact which is chosen to simulate the contact between the honeycombs and the top plate. It is reliable 
that the static and dynamic friction coefficients are set to 0.20 and 0.15 , respectively. In order to ensure accuracy of simulation, a convergence test is conducted to determine the suitable element size of the FE models. As shown in Fig. 4, the simulated SEA-Strain curves for different elements sizes are compared. It can be seen that SEA gradually converges when the element size reduces to $1 \mathrm{~mm} \times 1 \mathrm{~mm}$. Therefore, the element size of the $1 \mathrm{~mm} \times 1 \mathrm{~mm}$ is suitable for modeling these structures in the following studies.

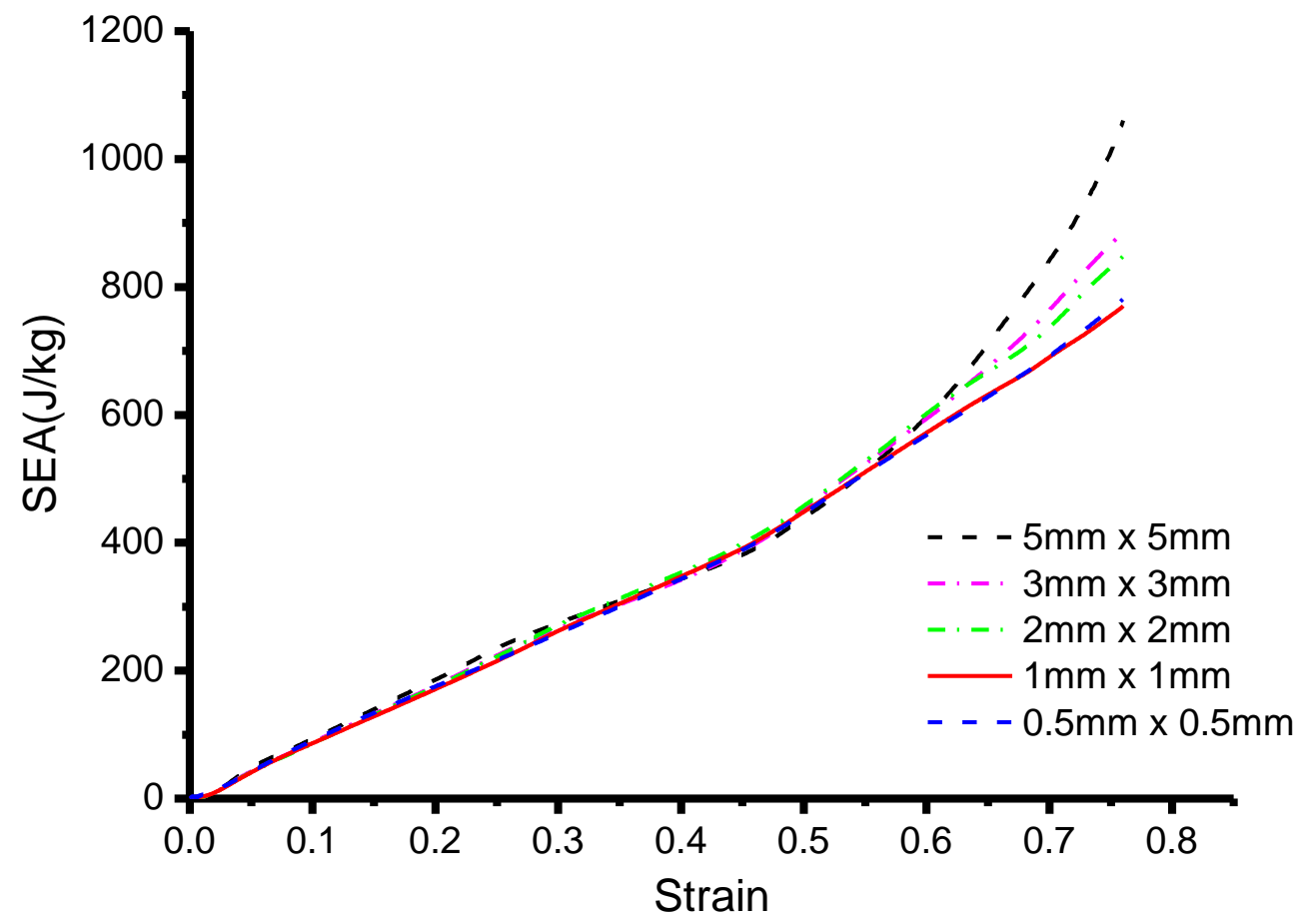

Fig. 4. Convergence test on RH with relative density of $5 \%$.

\subsection{Validation of the FE modeling}

To validate our simulated models, the benchmark between the experiment and the simulation has been conducted. The thickness of horizontal direction walls is $0.2 \mathrm{~mm}$ while the other walls are $0.1 \mathrm{~mm}$ in experiment [37]. The structure was compressed by a rigid plane with a constant velocity of $0.5 \mathrm{~mm} / \mathrm{min}$ in the vertical 
direction. As shown in Fig. 5 and Fig. 6, the deformation processes and the stress of the FE simulation and the experimental results are compared. It should be noticed that the deformations are in great agreement with the experiment results. Therefore, it has clearly validated that the FE models are able to predict the crushing performance of these hierarchical honeycombs.

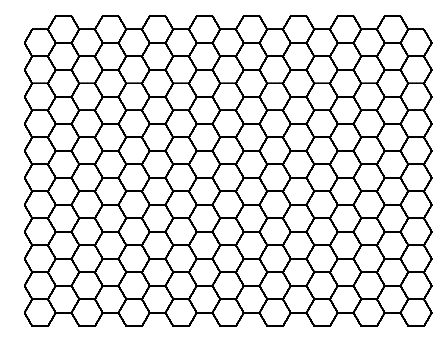

(a) $\varepsilon=0$

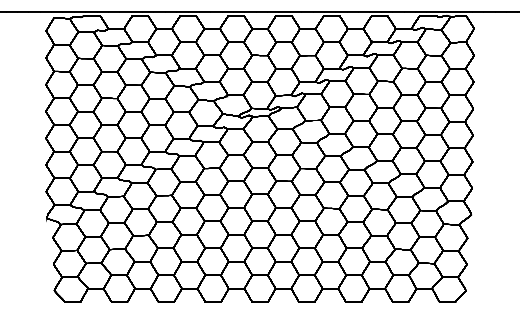

(b) $\varepsilon=0.119$

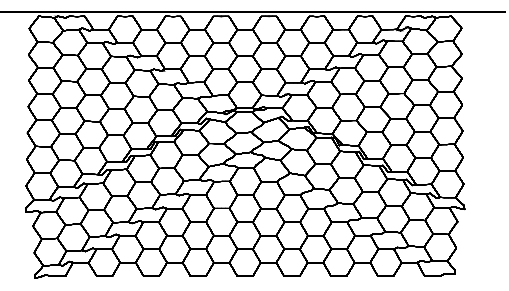

(c) $\varepsilon=0.209$

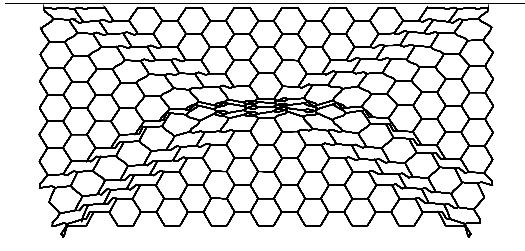

(d) $\varepsilon=0.349$

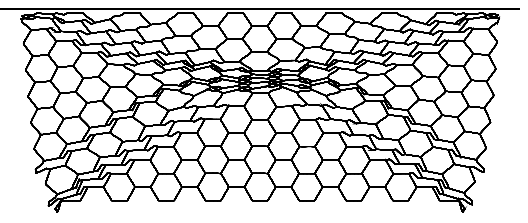

(e) $\varepsilon=0.438$

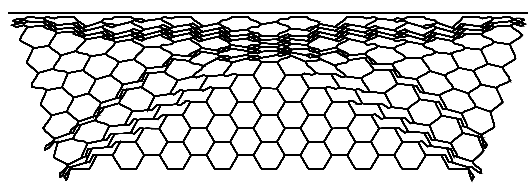

(f) $\varepsilon=0.558$

Fig. 5. Verification of deformation modes of finite element models compared to the experiment. [37] ( $\varepsilon$ is the nominal compressive strain) (a) $\varepsilon=0$. (b) $\varepsilon=0.119$. (c) $\varepsilon=0.209$. (d) $\varepsilon=0.349$. (e) $\varepsilon=0.438$.(f) $\varepsilon=0.558$.

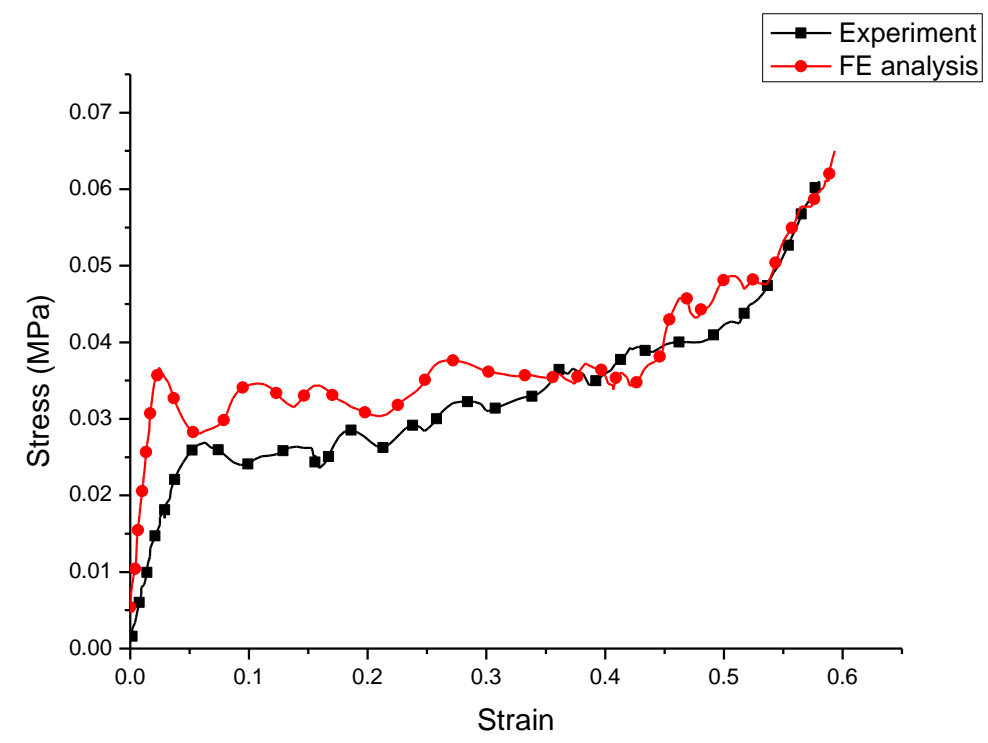

Fig. 6. Comparison of the stress obtained from the experiment [37] and the FE analysis. 


\section{Quasi-static collapse}

In this section, the quasi-static compression tests are conducted to analyze the crashworthiness of the honeycombs. A constant velocity of $1 \mathrm{~m} / \mathrm{s}$ is applied to the rigid plane to simulate quasi-static crushing. All honeycombs have the same relative density of $5 \%$. The thickness of RH, RHH and RHT are $0.48714 \mathrm{~mm}, 0.19287 \mathrm{~mm}$ and $0.07899 \mathrm{~mm}$. The masses of $\mathrm{RH}, \mathrm{RHH}$ and $\mathrm{RHT}$ are $0.07497 \mathrm{~kg}, 0.07618 \mathrm{~kg}$ and $0.07611 \mathrm{~kg}$, respectively.

\subsection{Uniaxial compression in the $x$ direction}

\subsubsection{Collapse mode}

A series of in-plane deformation configurations of RH, RHH and RHT along with different global strains in $x$ direction are shown in Fig. 7. As we can see, all honeycombs shrink slightly in the $y$ direction even when the deformation is small. The results obtained from Fig. 7(b) show that two types local bands are observed in all honeycombs. An obvious "V"-shape band occurs in the upper region of all models. The "I"-shape band appears mainly at the middle of RHH, while "I"-shape band of RH and RHT happens at the bottom. As the crushing proceeds, more cells near the deformed regions collapse, and "V"-shape bands and "I"-shape bands develop layer by layer because of the collapse propagation to other cells of these honeycombs. It should be noted that local densification takes place when the global strain reaches 0.37 in both RHH and RHT, which is presented in Fig. 7(d). In addition, the shapes of regular hexagonal substructure and triangular substructure of RHH and RHT in the horizontal cell walls remain unchanged while the unit cells collapse during the impact processes (see, Fig. 7(a)-(e)). Fig. 7(e) shows that the inclined walls of unit cells of RHH and RHT are basically damaged, and these hierarchical honeycombs are ready 
to further compact. The introduction of hierarchy significantly affects the deformation modes of re-entrant honeycombs.
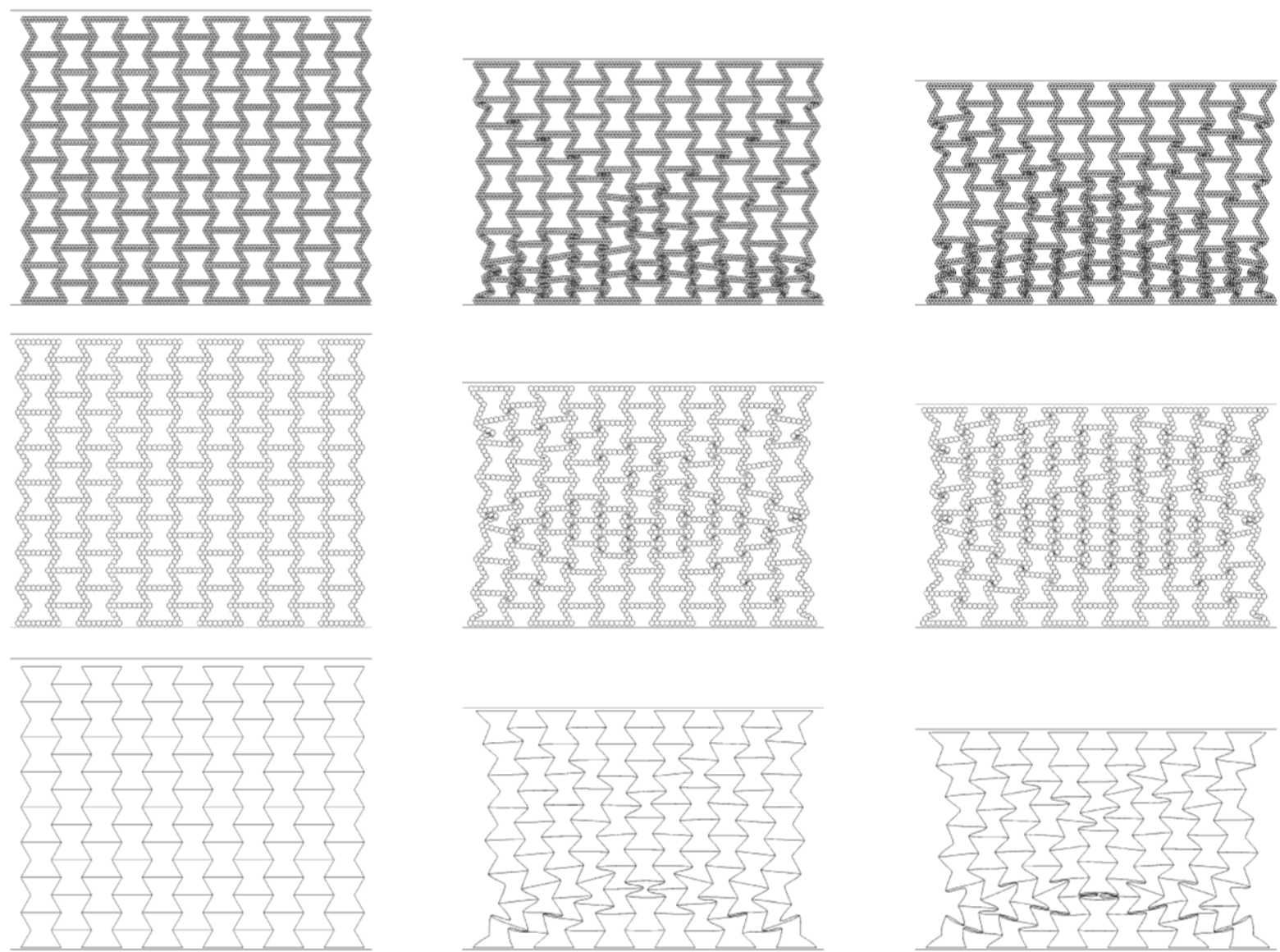

(a) $\varepsilon=0$

(b) $\varepsilon=0.17$

(c) $\varepsilon=0.26$
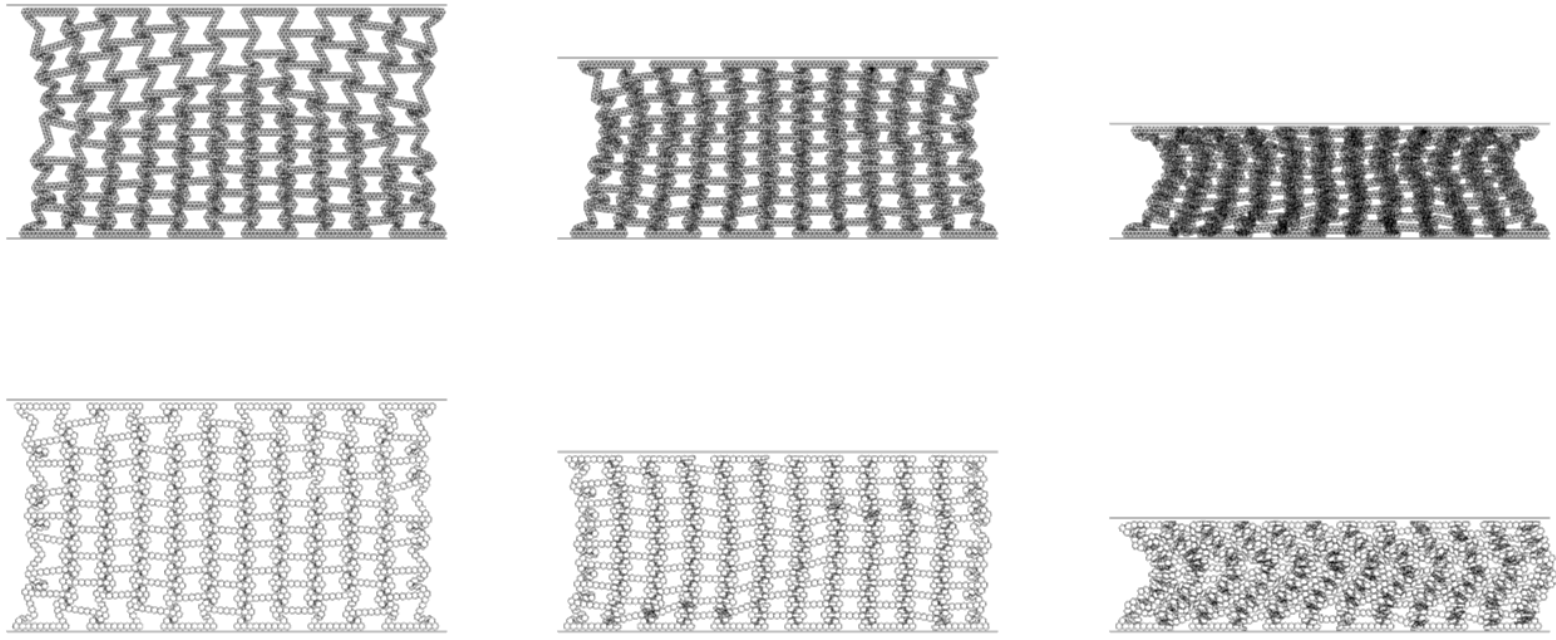


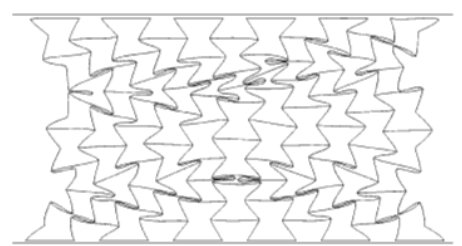

(d) $\varepsilon=0.37$

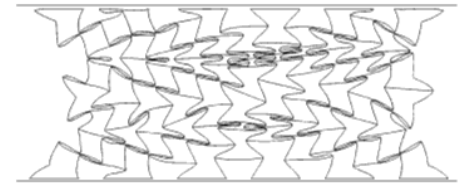

(e) $\varepsilon=0.52$

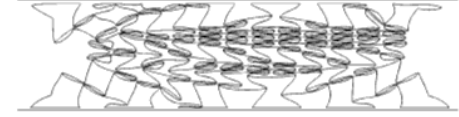

(f) $\varepsilon=0.72$

Fig. 7. Deformation processes of RH, RHH and RHT under quasi-static compression along the $x$ direction. (a) $\varepsilon=0$. (b) $\varepsilon=0.17$. (c) $\varepsilon=0.26$. (d) $\varepsilon=0.37$. (e) $\varepsilon=0.52$. (f) $\varepsilon=0.72$.

The typical local deformation patterns of RH, RHH and RHT are shown in Fig. 8 to further study the mechanical response of these honeycombs, respectively. A "< $\rightarrow$ " shape cell of the honeycombs is presented. In the compression process of the honeycombs, the substructures of horizontal direction do not suffer huge deformation, while the emblematical deformation cells for RH, RHH and RHT are "anchor" shape cell, "fork" shape cell and "six-side-cross" shape cell, respectively. All the representative cells rotate around the centers of the shape cells when these structures are crushed. It can be seen that the rotation degree of "six-side-cross" shape cell is much bigger than the rotations degrees of "anchor" and "fork" shape cells.
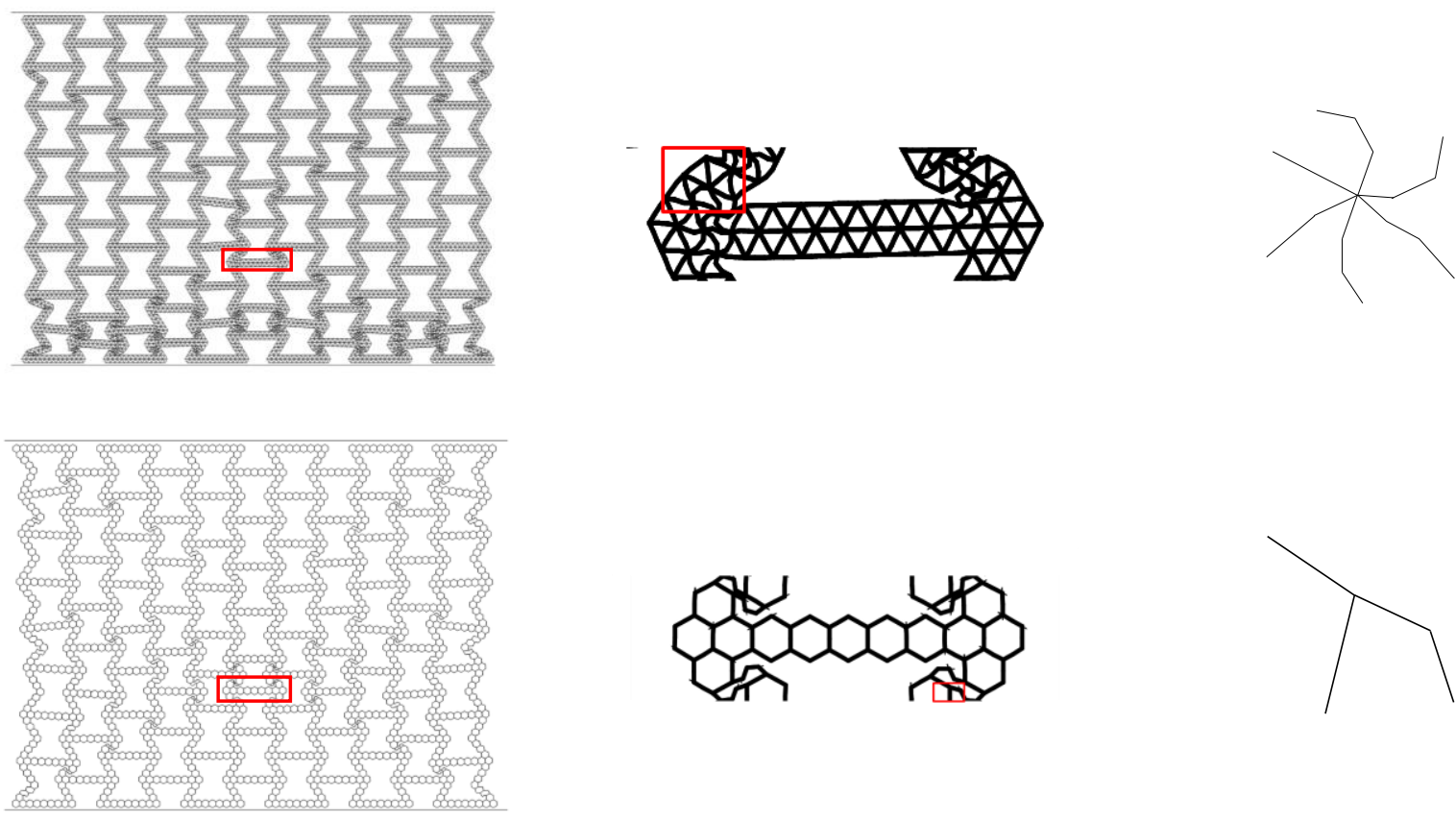

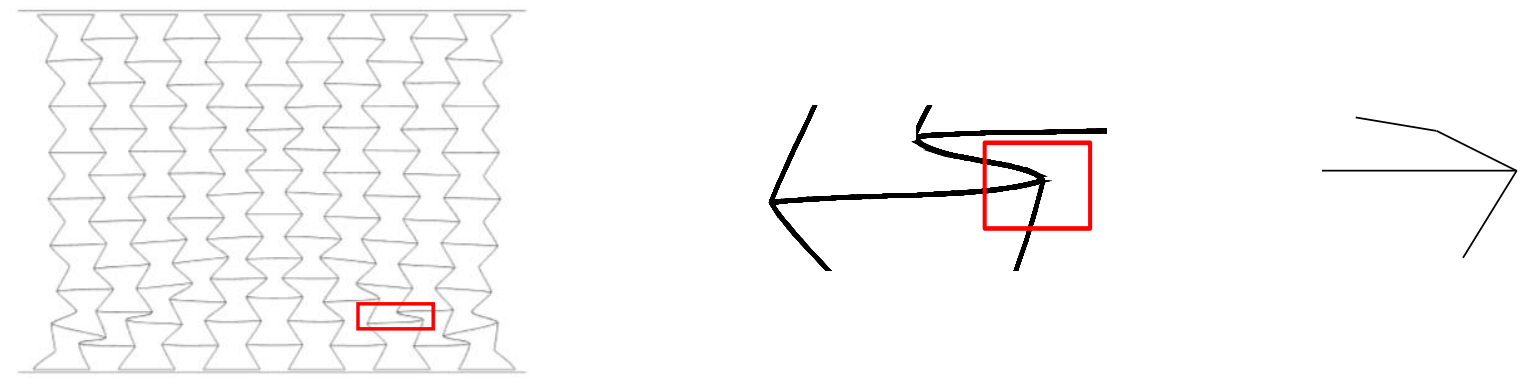

Fig. 8. A typical localized deformation patterns of these honeycombs with the strain of 0.1

\subsubsection{Collapse stress}

Based on the results from the simulated deformation modes, the stresses of RHT and RHH are obtained by numerical and analytical analysis. The stress is obtained by the ratio of the reaction force between the rigid plate and the specimen to the initial cross-sectional area of the honeycombs. The strain is derived from the ratio of the compression length of the honeycomb to its original length. Figure 9 presents the results obtain from the FE analysis of these honeycombs according to conversion. It is easy to point out that all curves present a common feature - a small peak stress at the initial strain and followed by a plateau phase of stress. The stresses of RHT and $\mathrm{RHH}$ are always higher than $\mathrm{RH}$ at various strains. What is interesting in this figure is that the stresses of RHT and RHH further rapidly increase as the strains increase while the stresses of RH keep a very slow growth. The results of FE analysis show that the stress value of RHT is $353 \%$ higher, RHH is $138 \%$ higher than RH at a strain of 0.25 . Specially, the RHT has a $2230 \%$ higher stress value compared to RH, and RHH has a $986 \%$ higher stress value than RH at a strain of 0.8 . These results indicate that the introduction of hierarchy has a lot of potential to improve the crushing performance. 


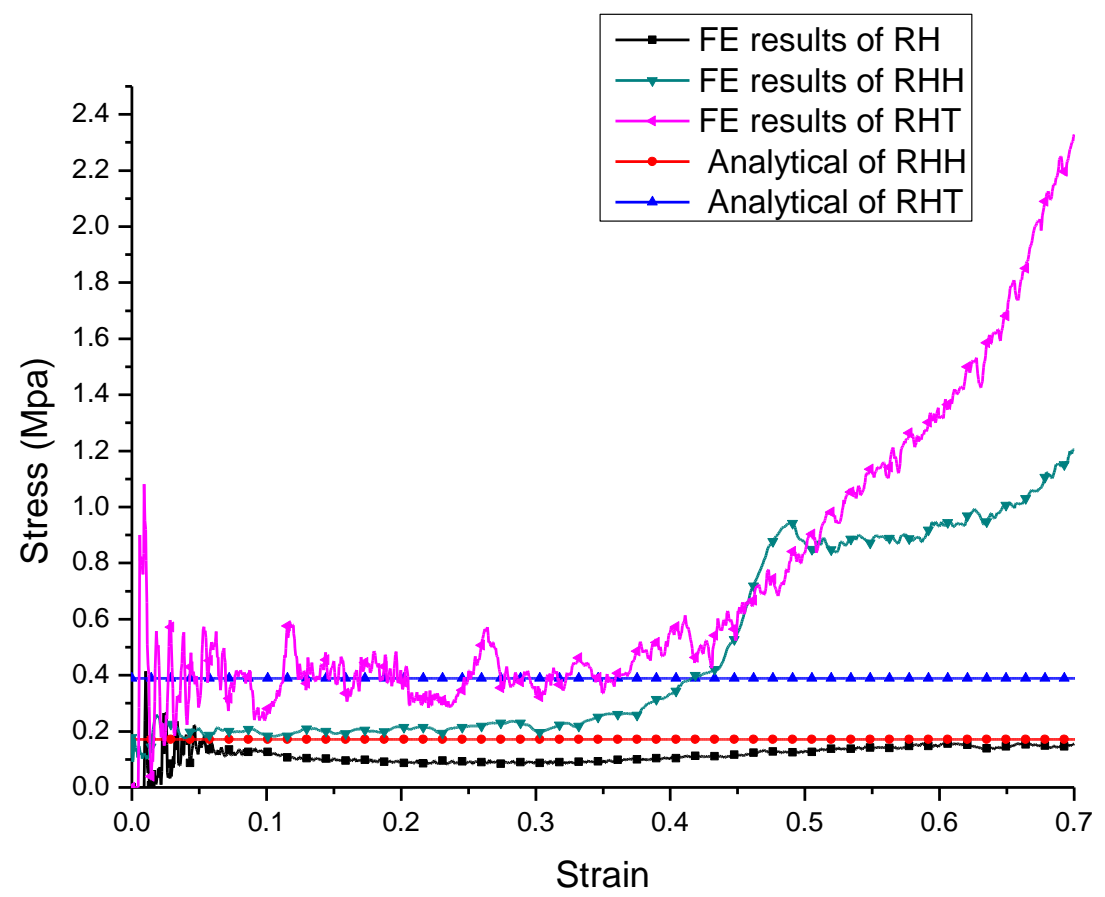

Fig. 9. Comparison of analytical results and FE results in $y$ direction with the relative density of $5 \%$.

The analytical results of plateau stress of RHT and RHH are derived by using the two-scale method [32]. The key of the method is that the global failure of hierarchical honeycombs is resulted from the collapse of the substructure. In this method, we only consider the deformation of localized deformation band which rotate and shorten in the process. As shown in Fig. 10, the representative unit of localized band which is a simplified model from Fig. 7 is presented. The black solid circles are plastic hinges which are the results from the rotation of red edges.

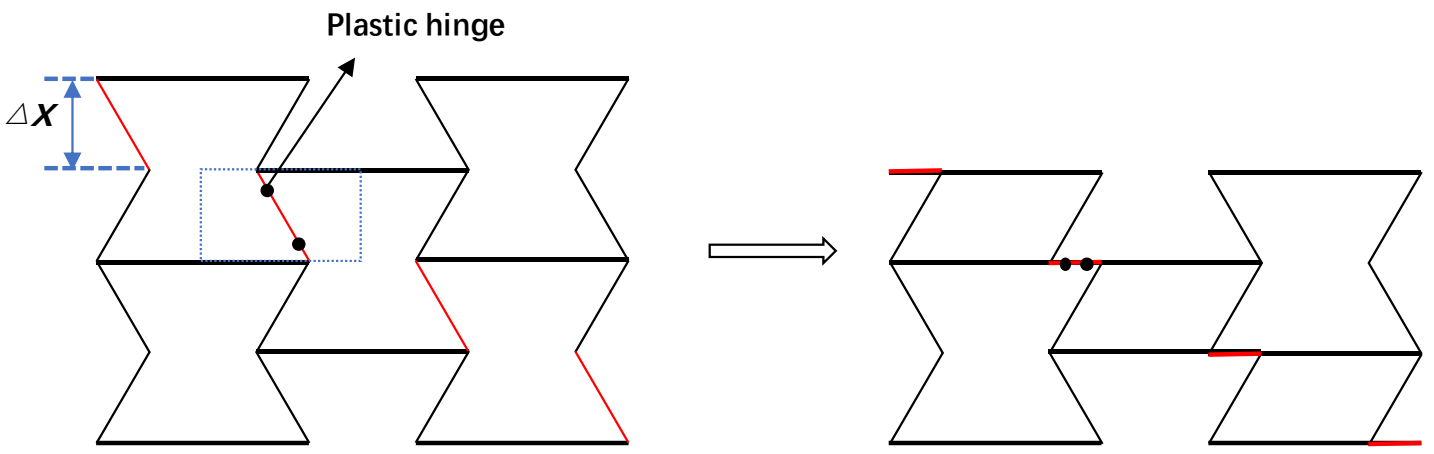

Fig. 10. Simplified representative unit of localized band of hierarchical honeycombs in the $x$ direction 
For RHT, the displacement caused by the external force is $\Delta x=\frac{\sqrt{3}}{2} N_{t 2} l_{t}$, and the external work is given by

$$
W_{e x t}=\frac{\sqrt{3}}{4} b l_{t}^{2}\left(2 N_{t 1}-N_{t 2}\right) N_{t 2} \sigma_{x t}
$$

where $\sigma_{x t}$ is the plateau stress of RHT in the $x$ direction.

The plastic dissipation caused by the two plastic hinges is calculated using $W_{h t}=2 M_{p t} \Delta \theta$, and the $M_{p t}$ is the bending moment of the cell wall $M_{p t}=\frac{3 b l_{t}^{2}}{4} \sigma_{0}^{t r i}$. Therefore,

$$
W_{h t}=\frac{\pi b l_{t}^{2}}{2} \sigma_{0}^{t r i}
$$

where $\sigma_{0}^{t r i}$ is the plateau stress of the equilateral triangular substructure, and it can be derived by [38]

$$
\sigma_{0}^{t r i}=C \bar{\rho}_{t r i}^{2} \sigma_{y s}
$$

the $\bar{\rho}_{t r i}$ is the relative density of equilateral triangular substructure, and $C=0.534$ is the constant which can be obtained by curve fitting the FE results.

Apart from the plastic dissipation caused by plastic hinges, the energy of the shortening of red edges should be considered. Correspondingly, the compressive dissipation can be derived by

$$
W_{d t}=\frac{\sqrt{3} N_{\mathrm{t} 2} b l_{t}^{2}}{2} \sigma_{0}^{t r i}
$$

According to this relation that $W_{e x t}=W_{h t}+W_{d t}$, the plateau stress of RHT can be calculated as 


$$
\sigma_{x t}=\frac{2\left(\pi+\sqrt{3} N_{\mathrm{t} 2}\right)}{\sqrt{3} N_{\mathrm{t} 2}\left(2 N_{\mathrm{t} 1}-N_{\mathrm{t} 2}\right)} \sigma_{0}^{t r i}
$$

For RHH, they have almost the same representative unit of localized band. The external work imposed on the representative unit of RHH is obtained as

$$
W_{e x h}=\frac{3 \sqrt{3}}{4} b l_{h}^{2}\left(2 N_{h 1}-N_{h 2}\right) N_{h 2} \sigma_{x h}
$$

where $\sigma_{x h}$ is the plateau stress of RHH in the $x$ direction.

The plastic dissipation of plastic hinges of RHH is acquired by $W_{h h}=2 M_{p h} \Delta \theta$,

$$
\begin{gathered}
M_{p h}=b l_{h}^{2} \sigma_{0}^{h e x} . \\
W_{h h}=\frac{2 \pi b l_{h}^{2}}{3} \sigma_{0}^{h e x}
\end{gathered}
$$

where $\sigma_{0}^{h e x}$ is the plateau stress of the regular hexagon substructure, and it can be obtained by [38]

$$
\sigma_{0}^{\text {hex }}=\frac{\mathrm{D}}{2} \bar{\rho}_{h e x}^{2} \sigma_{y s}
$$

the $\bar{\rho}_{\text {hex }}$ is the relative density of regular hexagon substructure, and $D / 2=0.5419$ is the constant which can be obtained by curve fitting the FE results.

Correspondingly, the compressive dissipation of RHH can be derived by

$$
W_{d h}=\sqrt{3} N_{h 2} b l_{h}^{2} \sigma_{0}^{h e x}
$$

According to this relation that $W_{e x h}=W_{h h}+W_{d h}$, the plateau stress of RHH can be calculated as

$$
\sigma_{x h}=\frac{4\left(2 \pi+3 \sqrt{3} N_{h 2}\right)}{9 \sqrt{3} N_{h 2}\left(2 N_{h 1}-N_{h 2}\right)} \sigma_{0}^{h e x}
$$

Figure 9 compares the plateau stress between the analytical predication and FE simulation of RHH and RHT in $x$ direction. It is apparent from the figure that the 
theoretical expression agrees pretty well with the FE results.

\subsubsection{Energy absorption}

For an energy absorption device, it is important to measure the ability of energy absorption with corresponding indexes. Here, the SEA and MCF are used to evaluate the impact performance. The SEA is one of the most important indexes to evaluate the performance of crashworthiness, and SEA is the main standard used to estimate the weight efficiency. As can be seen from Fig. 11, the SEA of all honeycombs increases significantly with the strain is raised. Notably the value of SEA of RHT is $292 \%$ higher than the value of $\mathrm{RH}$, and $\mathrm{RHH}$ is $105 \%$ higher than $\mathrm{RH}$ when the strain is 0.25 . Specifically, the RHH improves SEA by $430 \%$, and RHT further improves SEA by $782 \%$ above $\mathrm{RH}$ when the strain is 0.8 . It is apparent that the hierarchical designs can dramatically improve the ability of energy absorption. Figure 12 compares the results of MCF, which implies the higher energy absorption capability. There is a significant difference between these three structures. The MCF of RHT is always higher than RHH and the MCF of RHH is higher than RH. As shown in Fig. 12, the value of MCF of RHH is $108 \%$ higher than the value of RH while the value of RHT is $298 \%$ higher than RH when the strain is 0.25 . When the strain is 0.8 , the RHH improves MCF by $439 \%$, and RHT further improves MCF by $795 \%$ above RH. 


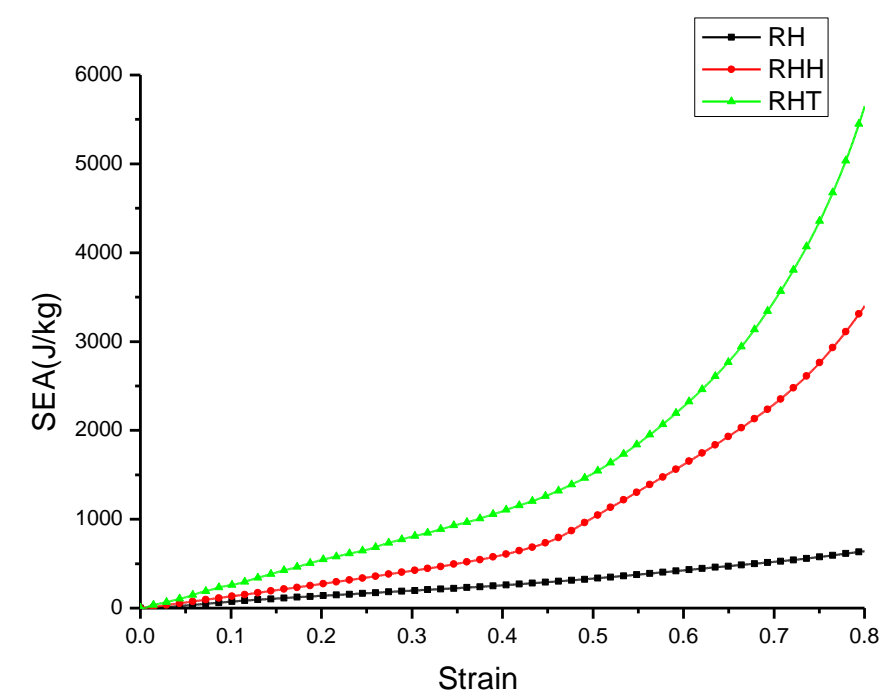

Fig. 11. SEA-strain curves of RH, RHH and RHT along the $x$ direction.

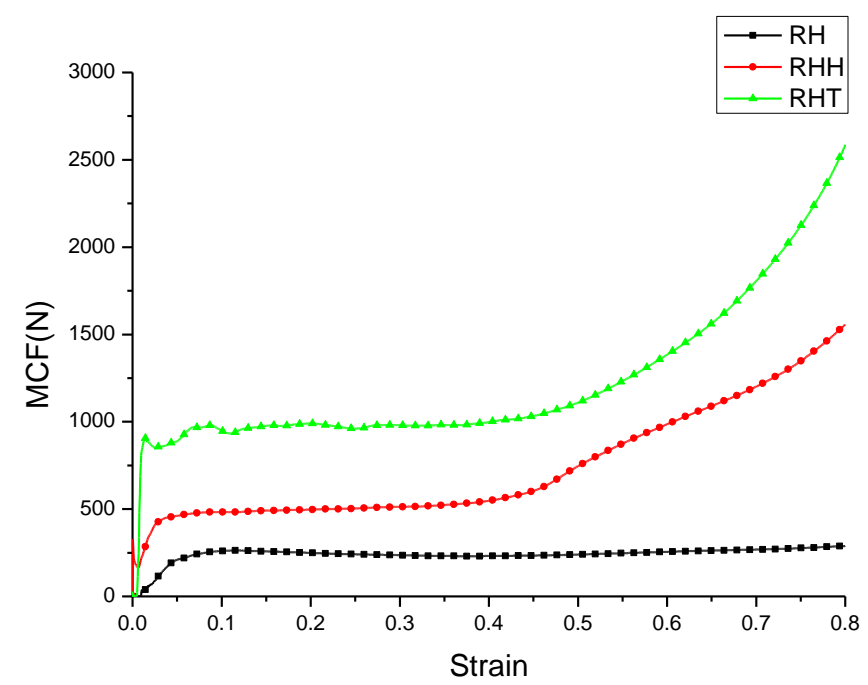

Fig. 12. MCF-strain curves of RH, RHH and RHT along the $x$ direction.

To further investigate the possible mechanisms responsible for the huge improvement in energy absorption, the stress plots before and after compression are compared in Fig. 13 to visualize the stress distribution of these honeycombs. It can be seen from Fig. 13 (a), (c) and (e) that RHT has more sub-honeycomb cells compared with RHH and RHT. To maintain the same relative density across all honeycombs, the thickness of RHT and RHH is much thinner than RH. As a result, the edges of hierarchical honeycombs become more deformable and create more plastic hinge 
during the collision, and thus contribute to superior energy absorption capability. As shown in Fig. 13 (b), (d) and (f), the sub-honeycomb of RHT has the biggest distortion.

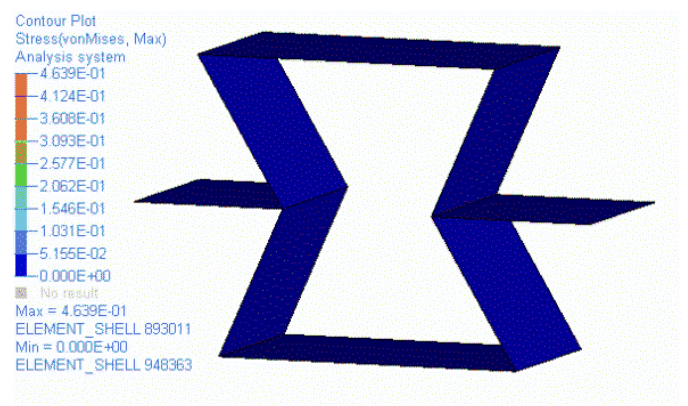

(a)

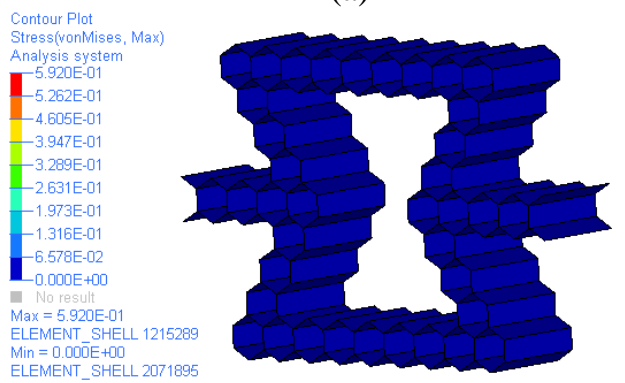

(c)

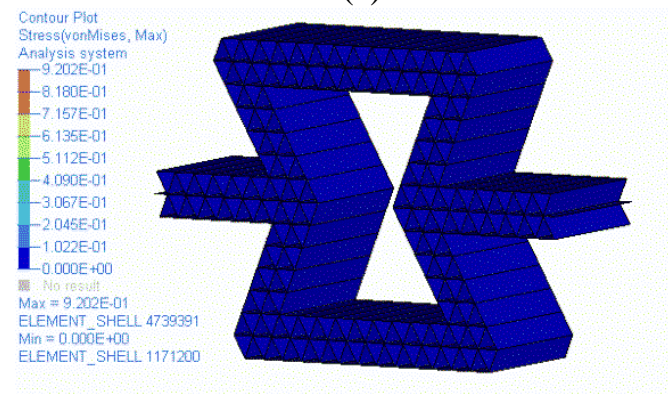

(e)

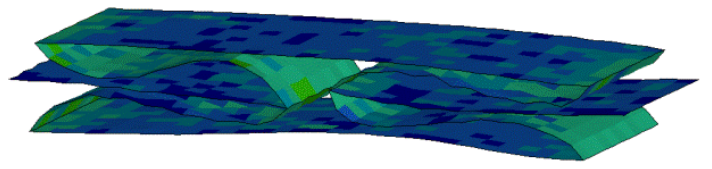

(b)

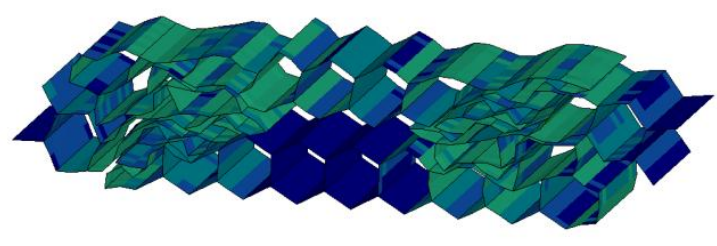

(d)

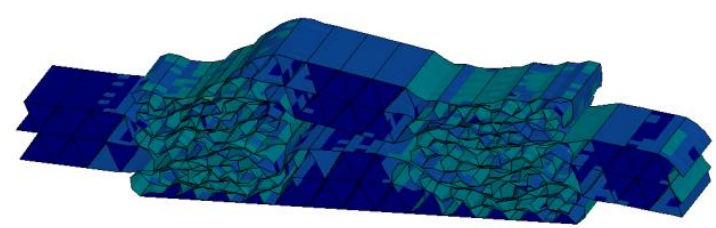

(f)

Fig. 13. Comparisons of stress diagram before and after compression. (a) Before compression of RH. (b) After compression of RH. (c) Before compression of RHH. (d) After compression of RHH. (e) Before compression of RHT. (f) After compression of RHT.

\subsubsection{Auxetic performance}

The variation of Poisson's ratio along the $x$ direction with strain is shown in Fig.

14. We define the ratio of the transverse extension strain to the longitudinal contraction strain as dynamic Poisson's ratio. The trend of dynamic Poisson's ratio of RHH and RH is almost the same. As can been seen from Fig. 14, the Poisson's ratio of RHH and RH is rapidly reduced to the minimum value and gradually increases with 
the increase of strain. Unlike the Poisson's ratio of $\mathrm{RHH}$ and $\mathrm{RH}$, the dynamic Poisson's ratio of RHT is slowly decreased. It should be pointed out that the dynamic Poisson's ratios of all honeycombs almost remain unchanged in the process of becoming compacted. Overall, these results indicate that the introduction of hierarchy on re-entrant honeycombs in this manner can affect the auxetic performance.

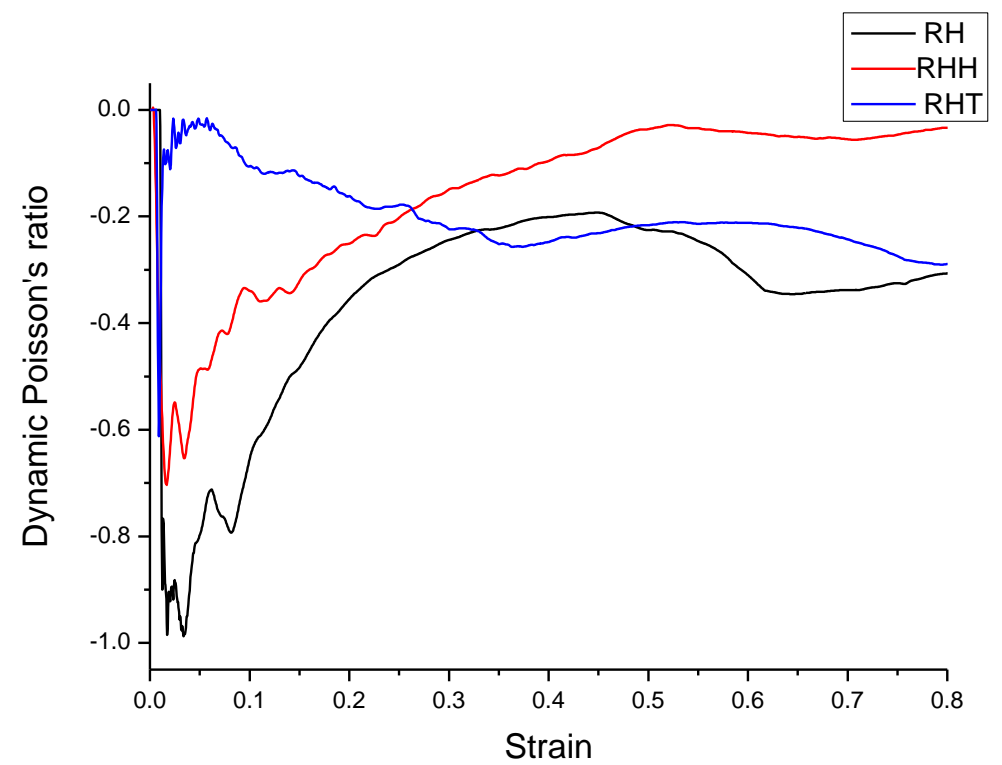

Fig. 14. Variation of Poisson's ratio of RH, RHH and RHT along the $x$ direction.

\subsection{Uniaxial compression in the y direction}

\subsubsection{Collapse mode}

Figure 15 presents the deformations processes of these honeycombs under the impact velocity of $1 \mathrm{~m} / \mathrm{s}$ along the $y$ direction. Similar to the results shown in Fig. 7 , all the honeycombs contract when they are compressed. The most striking observation to appear in the figure is that the honeycombs exhibit almost the same deformation pattern in the initial impact stage. As we can see, "I"- shape bands can be observed for RHT, RHH and RH, and the bands are local at the top and bottom of specimens. With the increase of the strain, the "I"- shape bands of these honeycombs continue to 
propagate from both ends. Interestingly, the unit cells of RHH and RHT away from the bands remain nearly unchanged, while the unit cells of RH undergone big changes. As the crushing proceeds, all honeycombs become compact.
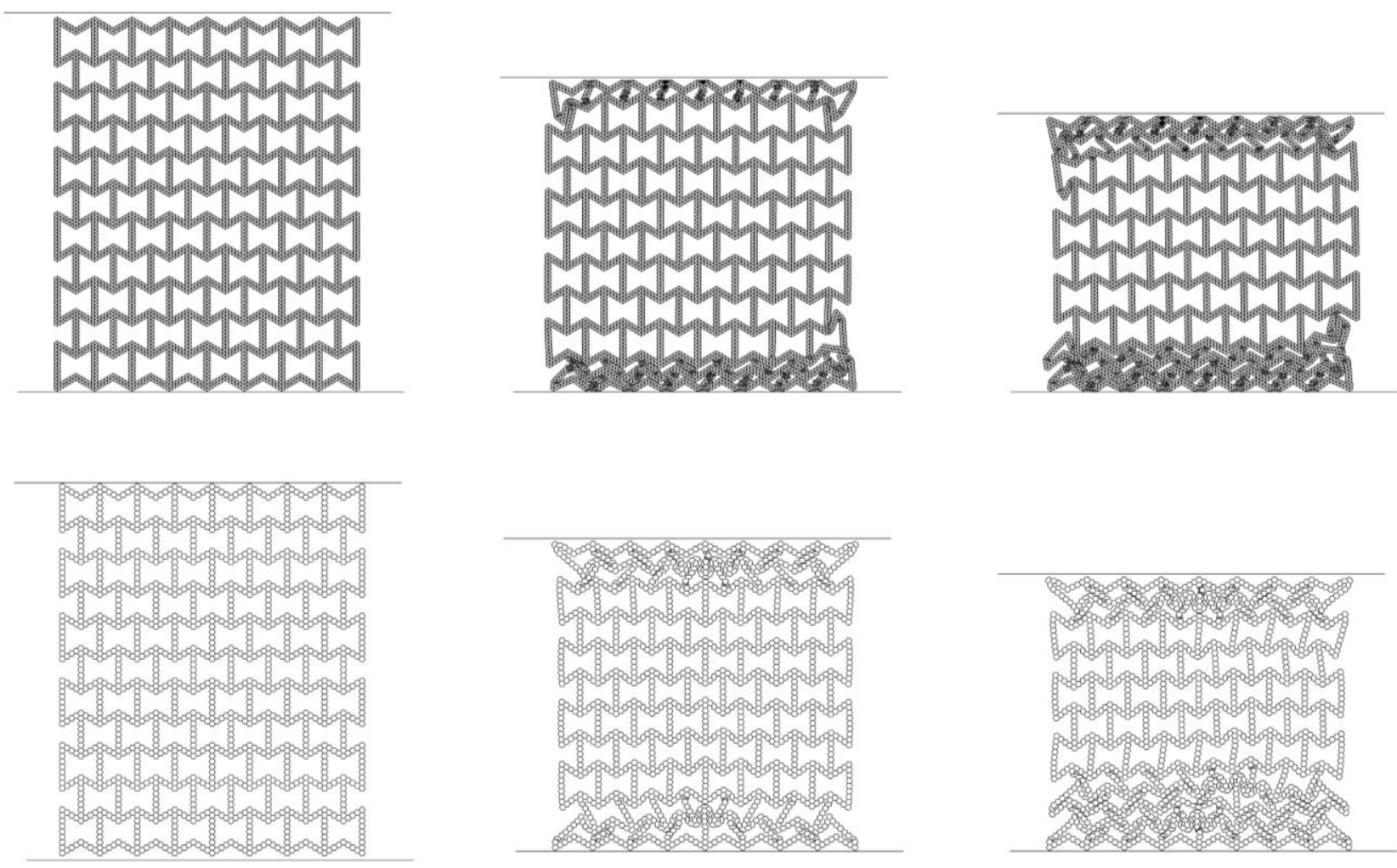

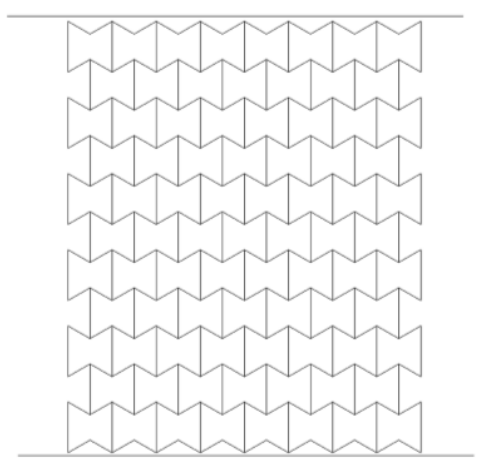

(a) $\varepsilon=0$

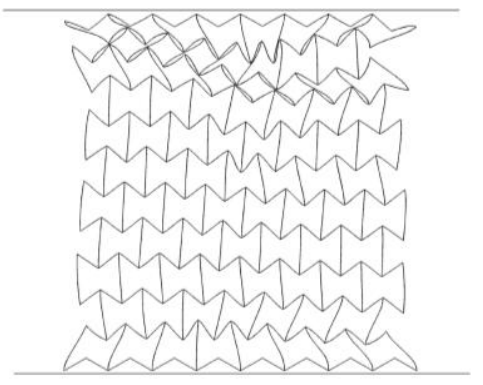

(b) $\varepsilon=0.17$

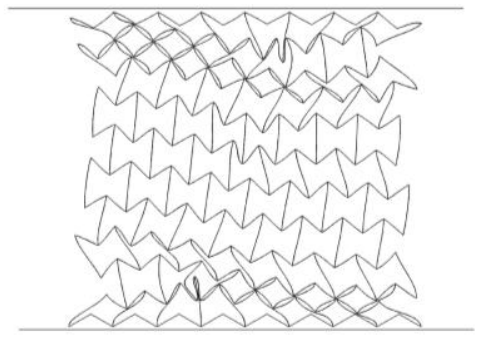

(c) $\varepsilon=0.27$ 

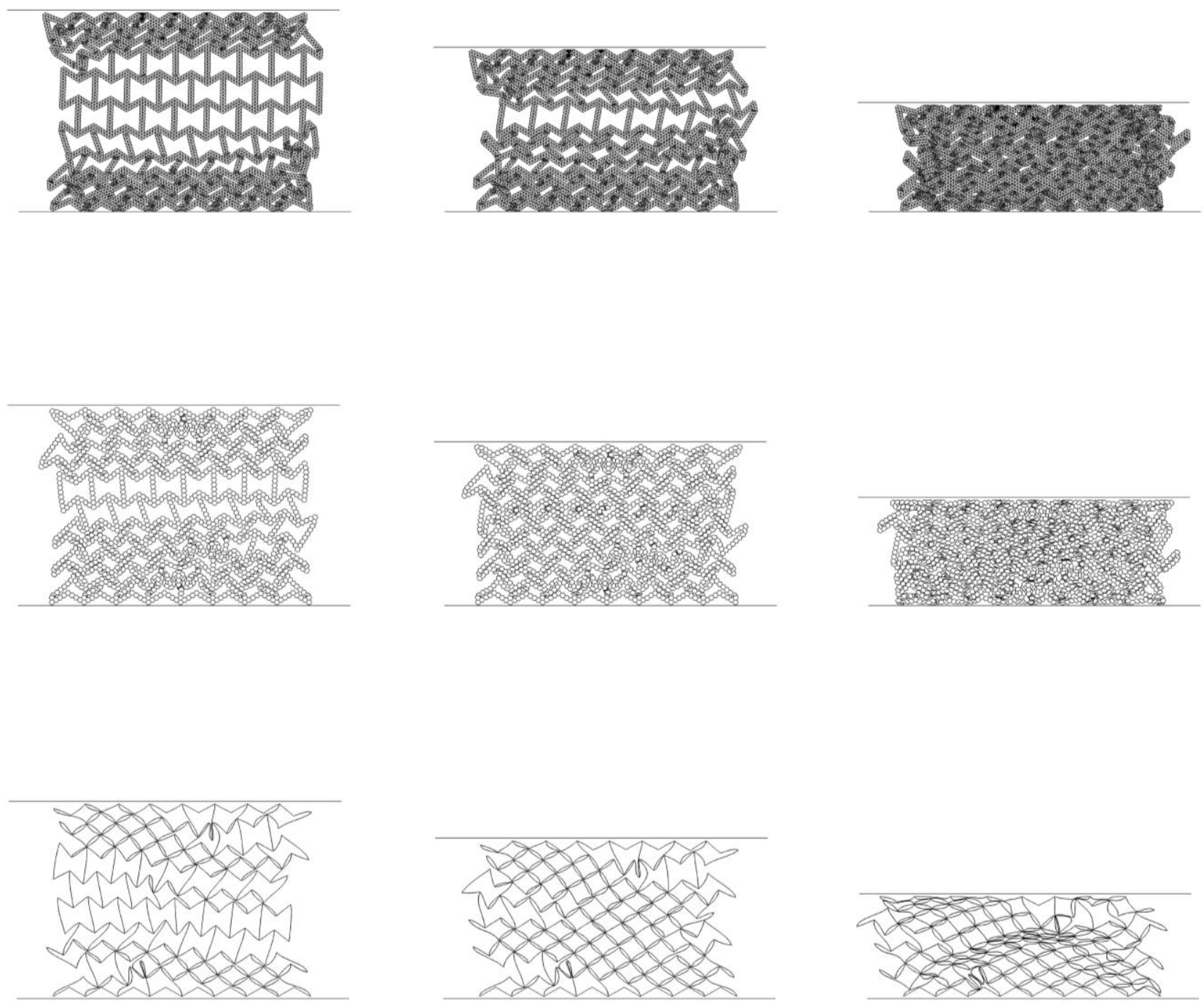

(d) $\varepsilon=0.38$

(e) $\varepsilon=0.51$

(f) $\varepsilon=0.68$

Fig. 15. Deformation processes of RH, RHH and RHT under quasi-static compression along the $y$ direction. (a) $\varepsilon=0$. (b) $\varepsilon=0.17$. (c) $\varepsilon=0.27$. (d) $\varepsilon=0.38$. (e) $\varepsilon=0.51$. (f) $\varepsilon=0.68$.

\subsubsection{Collapse stress}

As shown in Fig. 16, the stress-stain curves of the three honeycombs are compared. According to the response of stress, the re-entrant hierarchical honeycombs can be divided into three phases. Firstly, it is obvious that the initial elastic response of hierarchical honeycombs is detected when the strain is small, and the subsequent stress reaches the first peak (state I). Then, in the crushing process, the substructures 
of hierarchical honeycombs start to collapse, and the failure of the substructure increases the stiffness of unit cells which lead to the stress undulation (state II). Eventually, the hierarchical honeycombs become compact, and the stress increases dramatically (state III). It should be pointed out that the densification strain of RHH is slightly smaller than RHT. From the data in Fig. 16, the stresses of RHH and RHT are greatly larger than RH. The maximum stresses of RHT and RHH improve by $688 \%$ and $448 \%$ above $\mathrm{RH}$ at a strain of 0.8 , respectively. It is evident that the introduction of hierarchy has an important effect on stresses.

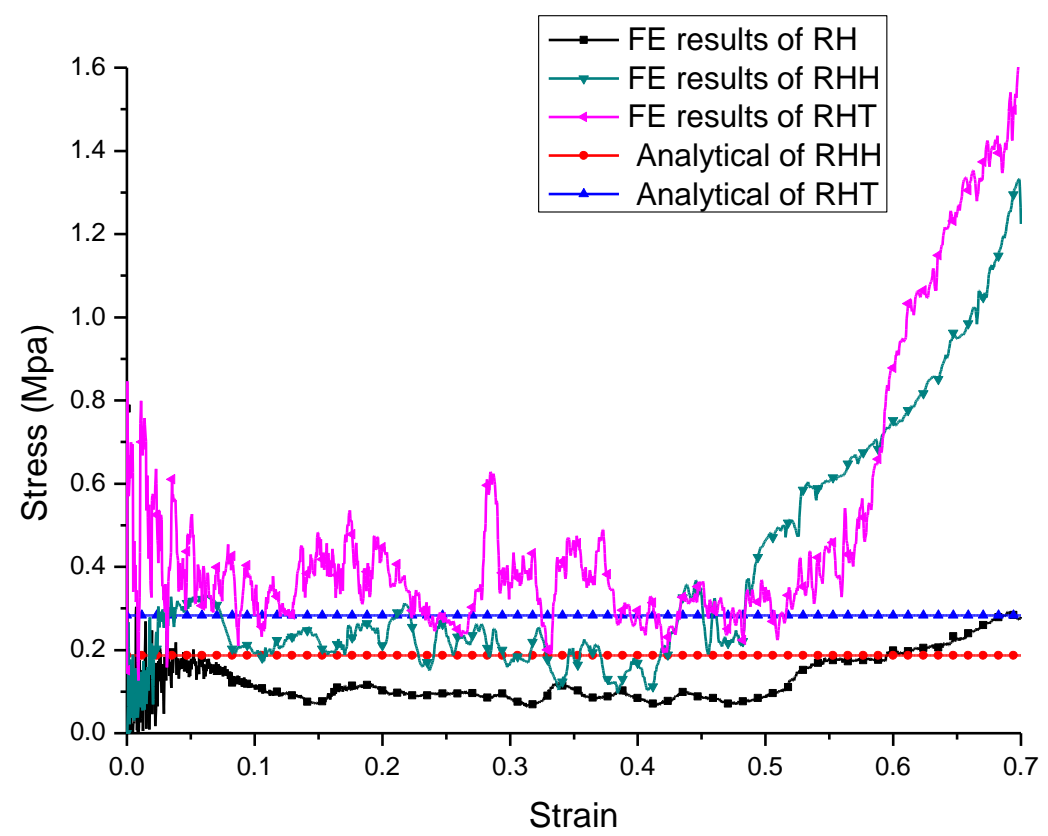

Fig. 16. Comparison of analytical results and FE results in $y$ direction with the relative density of $5 \%$.

Figure 15 compares the deformed patterns of RHH and RHT with relative density of 5\%. The simplified representative unit of RHH and RHT is provided in Fig. 17. The cell wall in the $y$ direction is rotated and shortened to the length of inclined walls. Based on the deformation modes in Fig. 17, the analytical results for RHT and RHH are derived by two-scale method. 

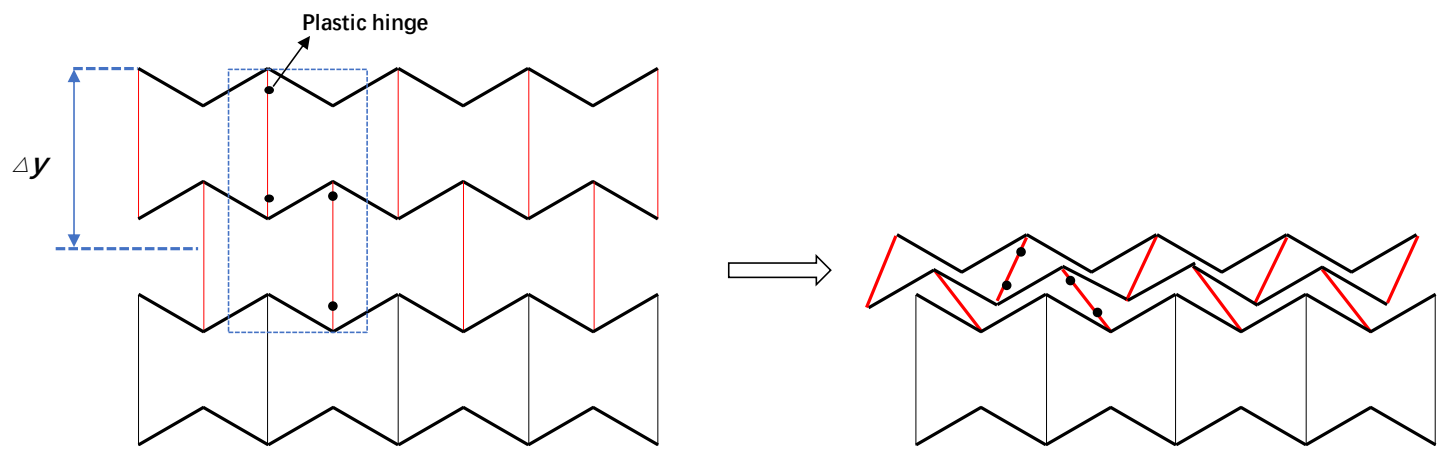

Fig. 17. Simplified representative unit of localized band of hierarchical honeycombs in the $y$ direction

For RHT, the displacement caused by the external force is $\Delta y=\left(2 N_{t 1}-\sqrt{3} N_{t 2}\right) l_{t}$, and the external work is given by

$$
W_{e x t}=\left(2 \sqrt{3} N_{t 1}-3 N_{t 2}\right) N_{t 2} b l_{t}^{2} \sigma_{y t}
$$

where $\sigma_{y t}$ is the plateau stress of RHT in the $y$ direction.

The plastic dissipation at four hinges is given by

$$
W_{h t}=\frac{\pi b l_{t}^{2}}{2} \sigma_{0}^{t r i}
$$

The compressive dissipation can be derived by

$$
W_{d t}=2 \sqrt{3}\left(N_{t 1}-N_{t 2}\right) b l_{t}^{2} \sigma_{0}^{t r i}
$$

According to this relation that $W_{e x t}=W_{h t}+W_{d t}$, the plateau stress of RHT in the $y$ direction can be calculated as

$$
\sigma_{y t}=\frac{\pi+4 \sqrt{3}\left(N_{t 1}-N_{t 2}\right)}{\left(4 \sqrt{3} N_{t 1}-6 N_{t 2}\right) N_{t 2}} \sigma_{0}^{t r i}
$$

For RHH, the external work is given by

$$
W_{e x h}=3\left(2 \sqrt{3} N_{h 1}-3 N_{h 2}\right) N_{h 2} b l_{h}^{2} \sigma_{y h}
$$

where $\sigma_{y h}$ is the plateau stress of RHH in the $y$ direction.

The plastic dissipation at four hinges is given by 
$W_{h h}=\frac{2 \pi b l_{h}^{2}}{3} \sigma_{0}^{h e x}$

The compressive dissipation can be derived by

$W_{d h}=4 \sqrt{3}\left(N_{h 1}-N_{h 2}\right) b l_{h}^{2} \sigma_{0}^{h e x}$

According to this relation that $W_{e x t}=W_{h t}+W_{d t}$, the plateau stress of RHT in the $y$ direction can be calculated as

$$
\sigma_{y h}=\frac{2 \pi+12 \sqrt{3}\left(N_{h 1}-N_{h 2}\right)}{9\left(2 \sqrt{3} N_{h 1}-3 N_{h 2}\right) N_{h 2}} \sigma_{0}^{h e x}
$$

Figure 16 presents the plateau stress of RHH and RHT in $y$ direction using the analytical solution and FE simulation. From the results in Fig. 16, it is seen that the analytical solutions agree very well with the FE results.

\subsubsection{Energy absorption}

Figure 18 presents the SEA of these honeycombs. With the crushing distance increases, SEA of all honeycombs keeps growth. The values of SEA of RHH and RHT are always higher than those of RH, which means that RHH and RHT can absorb more energy with the same mass. The SEA of RHT has semblable variation regular with RHH. When the strain is equal to 0.25 , the SEA of RHT and RHH is improved by $260 \%$ and $121 \%$ compared with RH. It is worth mentioning that the RHT improves the SEA value by $402 \%$, and RHH improves the SEA value by $300 \%$ at strain of 0.8 . Therefore, it clearly proves that the hierarchical characteristics can enormously increase the energy absorption efficiency. Figure 19 provides the results of MCF of honeycombs acquired from the analysis of FE simulation. The mean crushing force of RHH is higher than RH, however, the RHT shows the highest MCF. We note that the MCFs of all the honeycombs slightly go down after they reach the peak at lower 
strains. When the strain is equal to 0.25 , MCF of RHT and RHH is improved by $265 \%$ and $125 \%$ compared with $\mathrm{RH}$. As the strain is equal to 0.8 , the RHT and RHH improve the SEA values by $410 \%$, and $307 \%$, respectively.

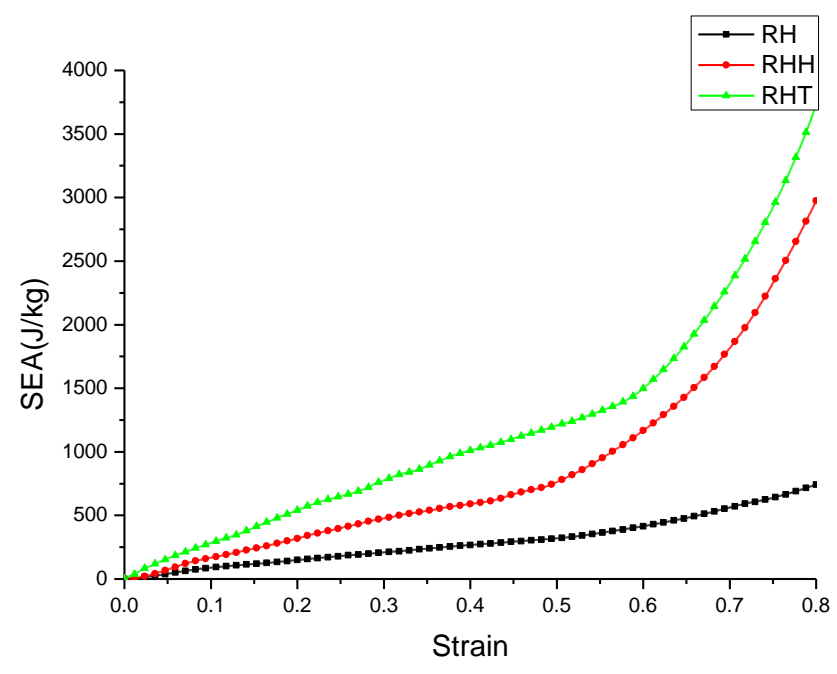

Fig. 18. SEA-strain curves of RH, RHH and RHT along the $y$ direction.

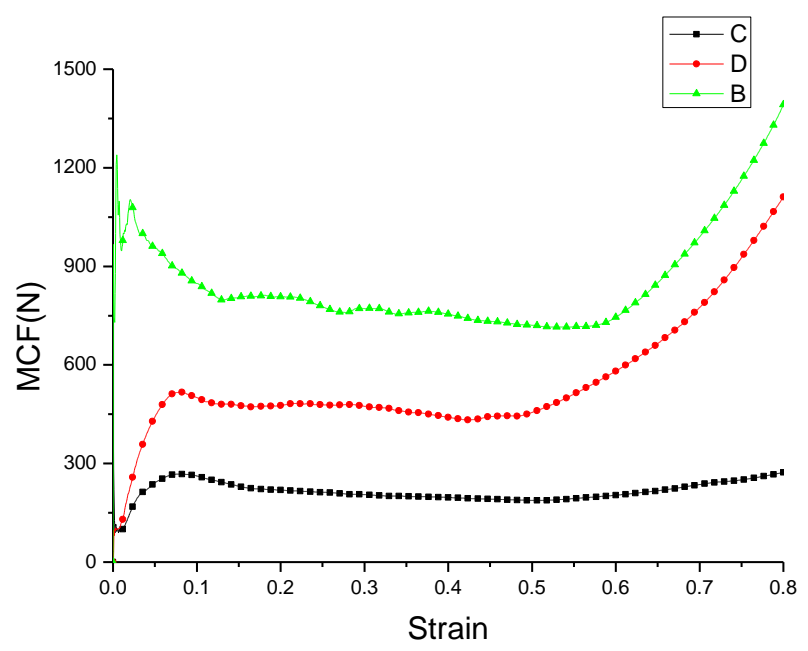

Fig. 19. MCF-strain curves of RH, RHH and RHT along the $y$ direction.

\subsubsection{Auxetic performance}

The variation of Poisson's ratio along the $y$ direction with strain is shown in Fig. 20. It is obvious that these honeycombs all have the properties of auxetic performance. 
Interestingly, the transverse contract of $\mathrm{RHH}$ and $\mathrm{RH}$ occurs mainly at the plateau region, while the transverse contract of RHT basically happens at the impact process.

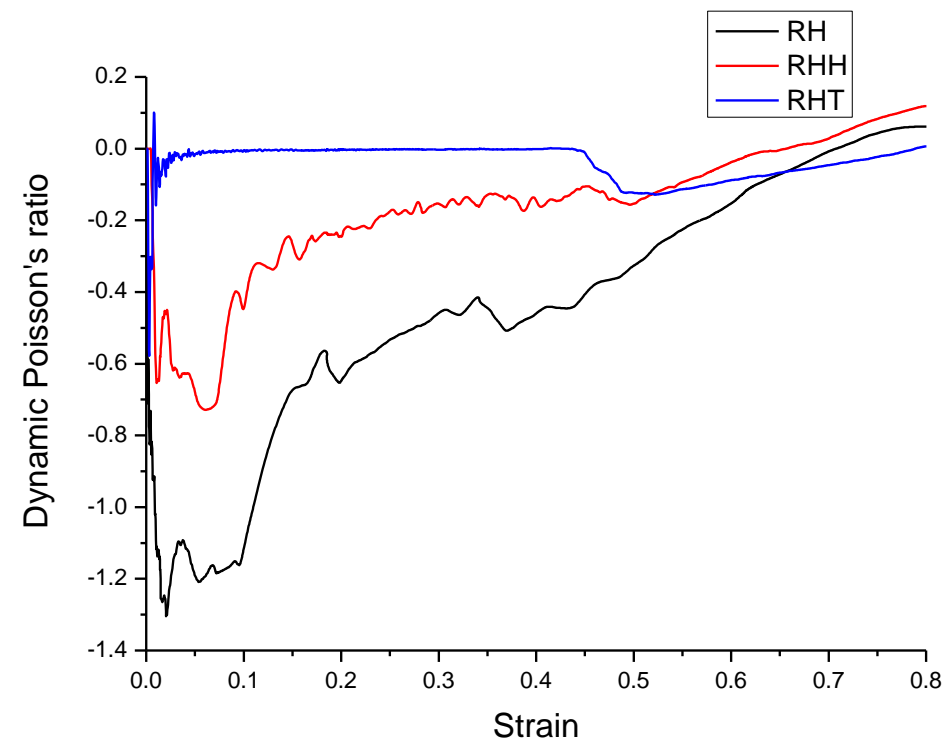

Fig. 20. Variation of Poisson's ratio of RH, RHH and RHT along the $x$ direction.

\section{Parametric studies of hierarchical honeycombs.}

The aim of this section is to investigate the effects of impact velocities and relative densities on energy absorption of these honeycombs. The change of relative density is accomplished by varying the wall thickness. Three different impact velocities $(5 \mathrm{~m} / \mathrm{s}, 20 \mathrm{~m} / \mathrm{s}, 50 \mathrm{~m} / \mathrm{s})$ are imposed on specimens to study the influence of crushing performance. The thickness of cell wall and the impact velocity are the two pivotal factors to determine the ability of absorbing energy.

\subsection{Effects of impact velocity}

Figure 21 compares the stresses of these honeycombs obtained from finite element method under different impact velocities. As the impact velocity increases, the stresses of RH, RHH and RHT increase at plateau regions. It is worth noting that the stresses of RHT are always the highest at different impact velocities compared to RH and RHT, which means the strongest energy absorption ability. As we can see, the 
stresses of RHH are larger than RH when the impact velocities are $5 \mathrm{~m} / \mathrm{s}$ and $20 \mathrm{~m} / \mathrm{s}$, while the values of RHH are nearly the same with RH under the impact velocity of $50 \mathrm{~m} / \mathrm{s}$. Figure 22 illustrates the variation of the SEA of these honeycombs under different impact velocities. Similar to the stress, the SEA of these honeycombs grows with the increase of strain. For the different impact velocities, the SEA of RHT is improved by $270 \%, 118 \%$ and $52 \%$ compared with $\mathrm{RH}$ at strain of 0.25 at velocity of $5 \mathrm{~m} / \mathrm{s}, 20 \mathrm{~m} / \mathrm{s}$ and $50 \mathrm{~m} / \mathrm{s}$, respectively. However, the SEA value of RHH is only improved by $60 \%$ and $15 \%$ compared with RH at velocity of $5 \mathrm{~m} / \mathrm{s}, 20 \mathrm{~m} / \mathrm{s}$ when strain is 0.25 , even when the velocity is $50 \mathrm{~m} / \mathrm{s}$, the value of SEA is lower than $\mathrm{RH}$. These findings confirm that the introduction of hierarchy can improve the capacity of energy absorption of the honeycombs. With the increase of the impact velocity, the effects of hierarchical honeycomb become weaker. Figure 23 shows the differences of MCF of these honeycombs under different impact velocities. Because of the effects of inertia, the MCF of all specimens shows higher value at low strains than plateau regime. It is noted that the MCF of RHT is still the highest under different impact velocities compared with RHH and RH. Compared with RH, the MCF of RHH is higher at $5 \mathrm{~m} / \mathrm{s}$ and $20 \mathrm{~m} / \mathrm{s}$, but lower at $50 \mathrm{~m} / \mathrm{s}$.

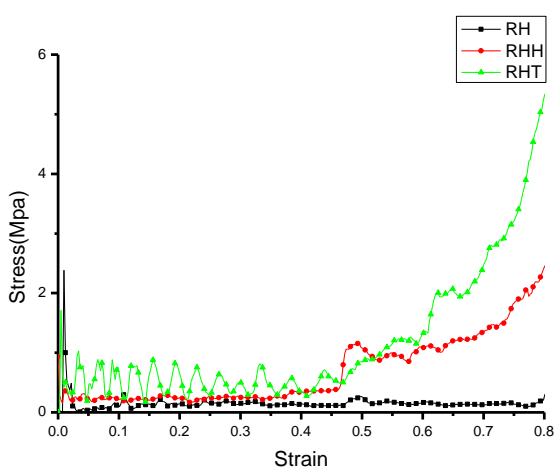

a

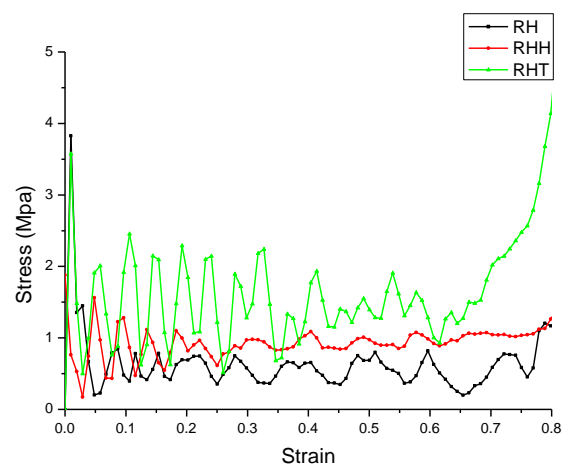

b

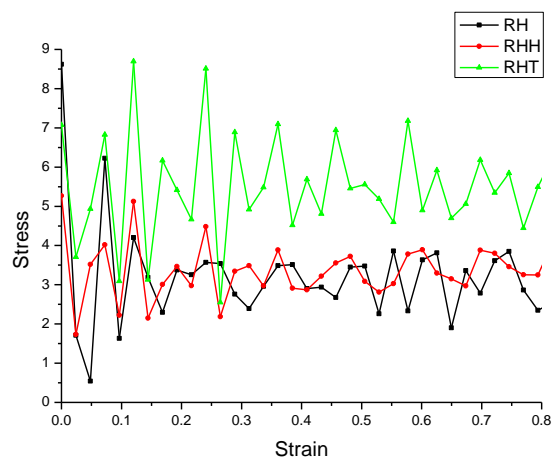

c

Fig. 21. Stress-strain curves of RH, RHH and RHT along the $x$ direction under different impact velocities.

(a) $5 \mathrm{~m} / \mathrm{s}$, (b) $20 \mathrm{~m} / \mathrm{s}$, (c) $50 \mathrm{~m} / \mathrm{s}$. 


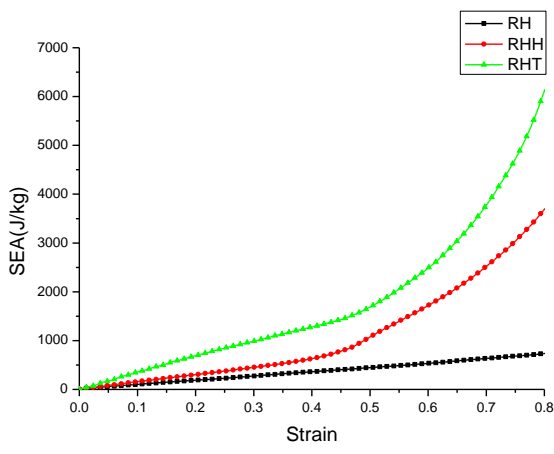

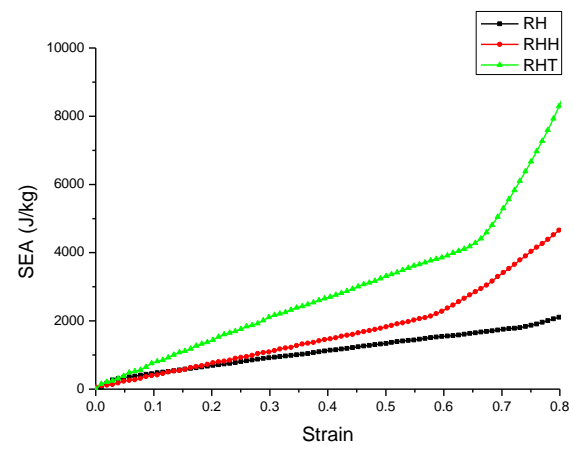

b

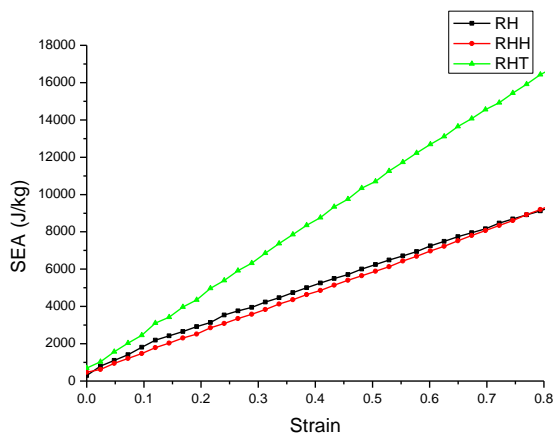

Fig. 22. SEA-strain curves of RH, RHH and RHT along the $x$ direction under different impact velocities. (a) $5 \mathrm{~m} / \mathrm{s}$, (b) $20 \mathrm{~m} / \mathrm{s}$, (c) $50 \mathrm{~m} / \mathrm{s}$.

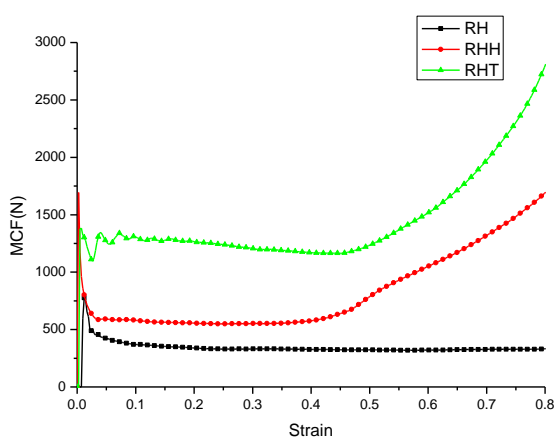

a

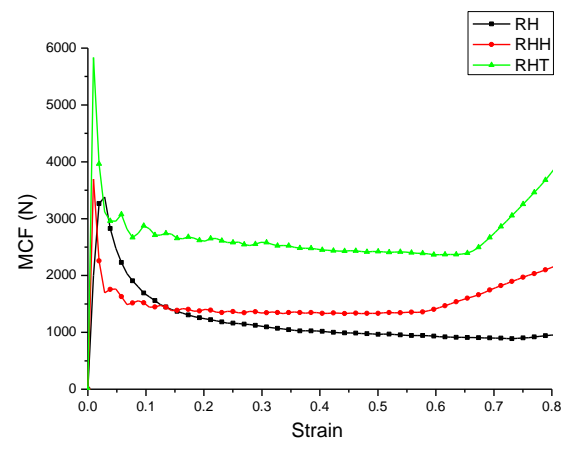

b

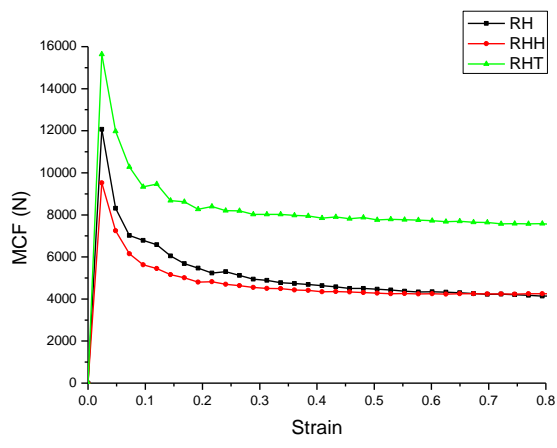

$\mathrm{c}$

Fig. 23. MCF-strain curves of RH, RHH and RHT along the $x$ direction under different impact velocities.

(a) $5 \mathrm{~m} / \mathrm{s}$, (b) $20 \mathrm{~m} / \mathrm{s}$, (c) $50 \mathrm{~m} / \mathrm{s}$.

Figure 24 compares the deformed shapes of honeycombs under different impact velocities at a strain of 0.193 . As we can see, the cell walls along $y$ direction of RHT and RHH almost remain unchanged with velocity of $5 \mathrm{~m} / \mathrm{s}$. When the hierarchical honeycombs are subjected to a high impact velocity, the cell walls along $y$ direction of RHT and RHH collapse and the "I" localized deformed bands are observed by all honeycombs. The "I" deformed bands propagate forward and show a feature of progressive row-by-row collapse feature, which means that the cell walls do not get much time to shrink transversely at a high impact velocity. 


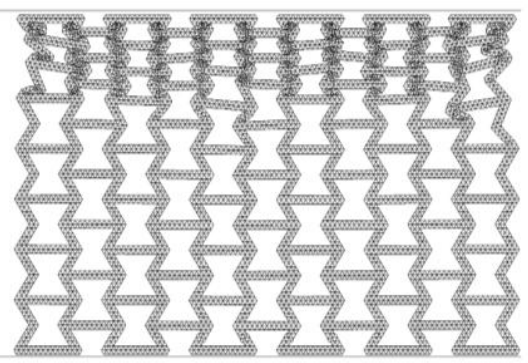

(a)

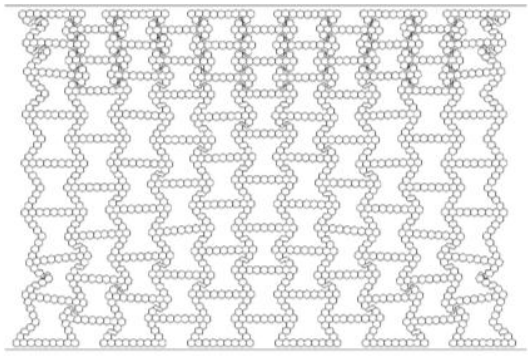

(c)

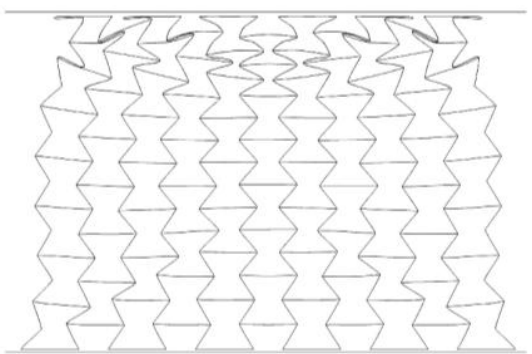

(e)

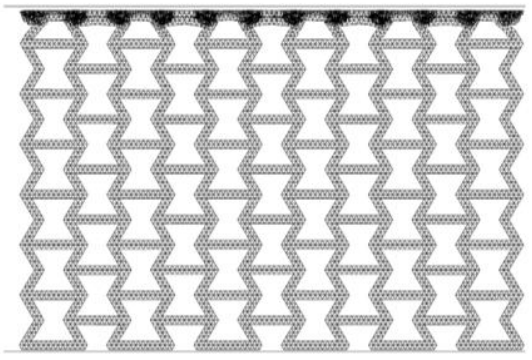

(b)

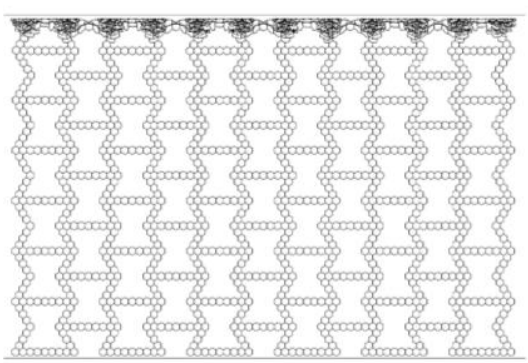

(d)

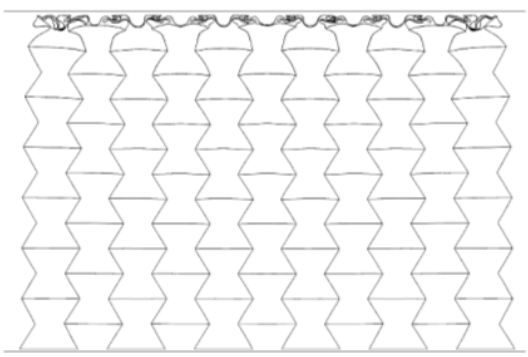

(f)

Fig. 24. Comparisons of deformed shapes under different impact velocities. (a) RHT with velocity of $5 \mathrm{~m} / \mathrm{s}$. (b) RHT with velocity of $50 \mathrm{~m} / \mathrm{s}$. (c) RHH with velocity of $5 \mathrm{~m} / \mathrm{s}$. (d) RHH with velocity of $50 \mathrm{~m} / \mathrm{s}$. (e) $\mathrm{RH}$ with velocity of $5 \mathrm{~m} / \mathrm{s}$. (f) $\mathrm{RH}$ with velocity of $50 \mathrm{~m} / \mathrm{s}$.

\subsection{Effect of the relative density}

In order to consider the influence of relative density on impact performance, we prepare several specimens of different relative densities. The change in relative density is done by varying the thickness of honeycombs. The thickness and weight assigned for each honeycomb is given in Table 1. Figure 25 displays the stresses of these honeycombs with different relative densities. It can be concluded that the stress increases with the cell wall thickness, and the variation trend of stress curves remains unchanged. For the hierarchical honeycombs, they have almost the same densification 
strain. Figs. 26 and 27 show how the SEA and MCF vary with the relative density. As can be seen from these figures, both SEA and MCF increase simultaneously with the wall thickness. Interestingly, the effects of relative density on MCF and stress are much greater than those on SEA. In terms of plateau regions, the stress and MCF are tended to be linear with relative density squared, while the SEA is linear with the relative density.

Table 1

Thickness and weight of honeycombs at different relative densities

\begin{tabular}{|c|c|c|c|c|}
\hline & & RH & RHH & RHT \\
\hline \multirow{2}{*}{$5 \%$} & $\begin{array}{l}\text { Thickness } \\
(\mathrm{mm})\end{array}$ & 0.48714 & 0.19287 & 0.07899 \\
\hline & $\begin{array}{c}\text { Weight } \\
(\mathrm{kg})\end{array}$ & 0.07497 & 0.07618 & 0.07611 \\
\hline \multirow{2}{*}{$10 \%$} & $\begin{array}{l}\text { Thickness } \\
(\mathrm{mm})\end{array}$ & 0.97428 & 0.38574 & 0.15799 \\
\hline & $\begin{array}{l}\text { Weight } \\
(\mathrm{kg})\end{array}$ & 0.14994 & 0.15236 & 0.15222 \\
\hline \multirow{2}{*}{$20 \%$} & $\begin{array}{l}\text { Thickness } \\
(\mathrm{mm})\end{array}$ & 1.94856 & 0.77149 & 0.31598 \\
\hline & $\begin{array}{c}\text { Weight } \\
(\mathrm{kg})\end{array}$ & 0.2998 & 0.30472 & 0.30444 \\
\hline
\end{tabular}

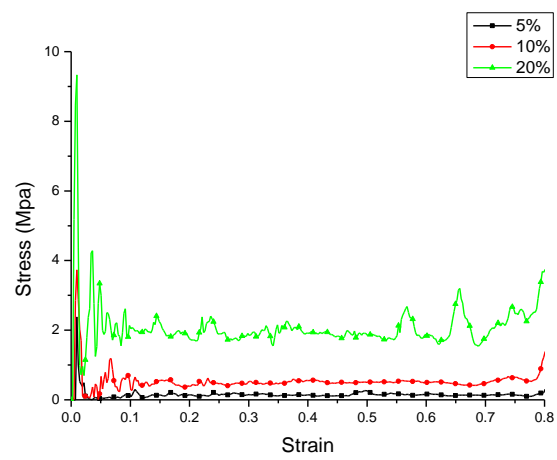

$\mathrm{a}$

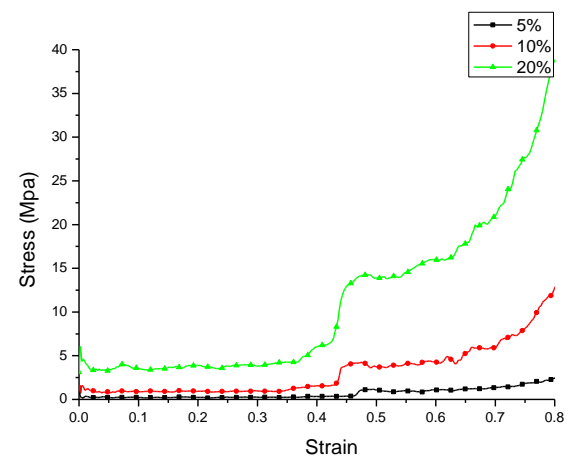

b

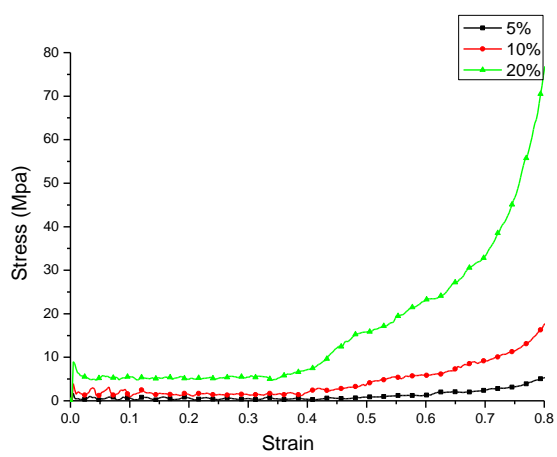

c

Fig. 25. Comparison of Stresses of honeycombs with different relative densities. (a) RH, (b) RHH, (c) RHT. 


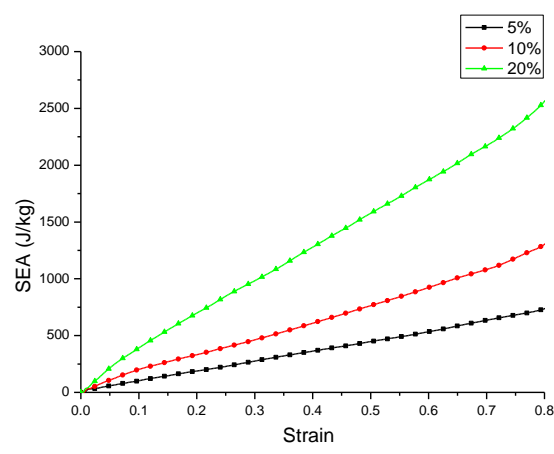

a

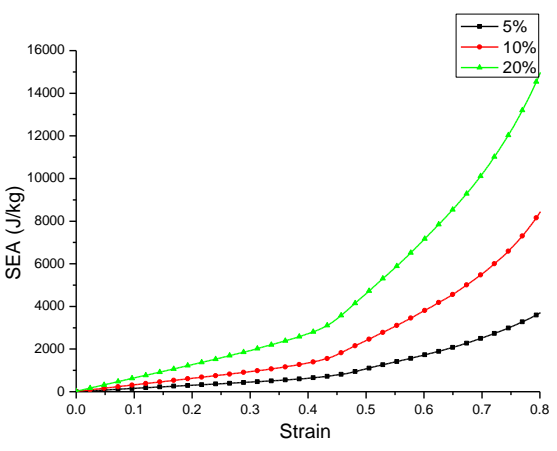

b

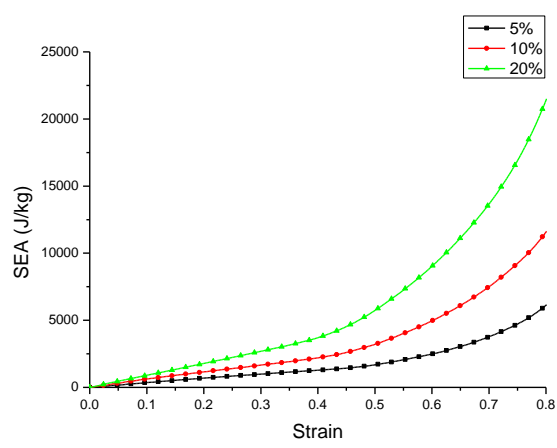

Fig. 26. Comparison of SEAs of honeycombs with different relative densities. (a) RH, (b) RHH, (c) RHT.

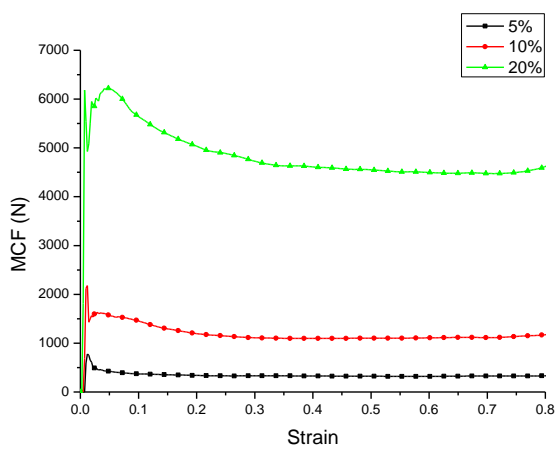

a

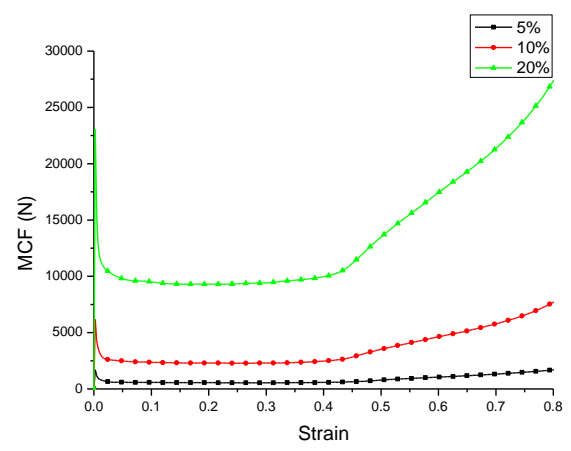

b

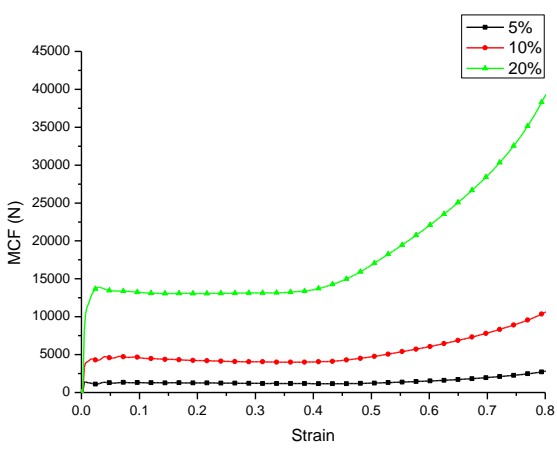

$\mathrm{c}$

Fig. 27. Comparison of MCFs of honeycombs with different relative densities. (a) RH, (b) RHH, (c) RHT.

\section{Concluding remarks}

The in-plane crashworthiness of re-entrant hierarchical honeycombs has been systematically investigated in this work. We have discussed the collapse mode and energy absorption performance of hierarchical honeycombs under quasi-static compression in the $x$ and $y$ directions. As a further comparsion, we have also conducted the parametric studies to explore the effects of impact velocity and relative density on the energy absorption performance. Based on the results among these three honeycombs, the following conclusions can be drawn:

(1) In the quasi-static compression, the two-scale method is used to predict the plateau stress of RHH and RHT in $x$ and $y$ directions. The FE results agree very well with theoretical solutions. 
(2) In the quasi-static compression, the proposed re-entrant hierarchical honeycombs are much better energy absorption devices compared with RH. The stress value of RHT is $353 \%$ higher, and RHH is $138 \%$ higher than RH at plateau regions. The value of SEA of RHT is $292 \%$ higher than the value of RH, and RHH is $105 \%$ higher than RH. The value of SEA of RHT is $292 \%$ higher than the value of RH, and $\mathrm{RHH}$ is $105 \%$ higher than $\mathrm{RH}$ in the $x$ direction.

(3) With the increase of the impact velocity, the improvement of energy absorption capacity of hierarchical honeycomb decreases. Under the low and medium impact velocities, the RHT and RHH have the much better energy absorption ability compared with RH. When subjected to the high impact velocity, RHT still has the best crashworthiness performance, while RHT performs almost the same with RH.

(4) The influences of relative density on stress, SEA and MCF are different. The effects of relative density on MCF and stress are much greater than that on SEA.

\section{Statement about the feasibility of the experimental plan}

This section presents the details of experimental plan, and the manufacture of honeycombs and experimental methods are discussed. The previous study has already given advice on the manufacture of auxetic honeycomb [16]. The regular auxetic honeycomb are made by folding the aluminum AA6061 sheets manually to the required profile, then the sheets were bonded using epoxy resin with adjacent layers facing oppositely. The fabrication of hierarchical honeycomb was provided by Fang et al. [28]. The specimen is manufactured by employing the wire cut using the electrical discharge machining process to cut an AA6061 block. The fabrication of the proposed 
hierarchical honeycombs (RHT and RHH) in this paper will adopt the wire cut to form specimens. The sample manufacturing steps are as follows: (a) a specified height aluminum block is cut from along aluminum; (b) the block is drilled to make holes in order to remove the material which is not needed; (c) threading the wire in the holes and performing the wire-cut operation.

The compression tests will carry out through an upper smooth indenter in the INSTRON=5984 test machine which can provide a standard method of acquiring the compressive strength.

\section{Acknowledgments}

This work was supported by the Foundation for innovative Research Groups of the National Natural Science Foundation OF China (Grant No.51621004).

\section{References}

[1] Gibson LJ, Ashby MF. Cellular solids: structure and properties: Cambridge university press, 1999.

[2] Lu TJ. Heat transfer efficiency of metal honeycombs. International Journal of Heat and Mass Transfer. 1998;42:2031-40.

[3] Wierzbicki T. Crushing analysis of metal honeycombs. International Journal of Impact Engineering. 1983;1:157-74.

[4] Hu LL, Yu TX. Mechanical behavior of hexagonal honeycombs under low-velocity impact - Theory and simulations. International Journal of Solids \& Structures. 2013;50:3152-65.

[5] Cricrì G, Perrella M, Calì C. Honeycomb failure processes under in-plane loading. Composites Part B: Engineering. 2013;45:1079-90.

[6] Papka SD, Kyriakides S. Experiments and full-scale numerical simulations of in-plane crushing of a honeycomb. Acta Materialia. 1998;46:2765-76.

[7] Ruan D, Lu G, Wang B, Yu TX. In-plane dynamic crushing of honeycombs — a finite element study. International Journal of Impact Engineering. 2003;28:161-82.

[8] Wu E, Jiang W-S. Axial crush of metallic honeycombs. International Journal of Impact Engineering. 1997;19:439-56.

[9] Mukhopadhyay T, Adhikari S, Alu A. Probing the frequency-dependent elastic moduli of lattice materials. Acta Materialia. 2019;165:654-65.

[10] Yuan L, Shi H, Ma J, You Z. Quasi-static impact of origami crash boxes with various profiles. Thin-Walled Structures. 2019;141:435-46.

[11] Li Y, You Z. Open-section origami beams for energy absorption. International Journal of Mechanical Sciences. 2019;157-158:741-57.

[12] Evans KE, Alderson KL. Auxetic materials: the positive side of being negative. Engineering Science \& Education Journal. 2000;9:148-54.

[13] Liu W, Wang N, Tao L, Lin Z. In-plane dynamic crushing of re-entrant auxetic cellular structure. Materials \& Design. 2016;100:84-91.

[14] Zhang X-C, An L-Q, Ding H-M, Zhu X-Y, El-Rich M. The influence of cell micro-structure on the in-plane dynamic crushing of honeycombs with negative Poisson's ratio. Journal of Sandwich Structures \& Materials. 2015;17:26-55.

[15] Jin X, Wang Z, Ning J, Xiao G, Liu E, Shu X. Dynamic response of sandwich structures with graded auxetic honeycomb cores under blast loading. Composites Part B Engineering. 
2016;106:206-17.

[16] Qi C, Remennikov A, Pei LZ, Yang S, Yu ZH, Ngo TD. Impact and close-in blast response of auxetic honeycomb-cored sandwich panels: experimental tests and numerical simulations. Composite Structures. 2017;180.

[17] Lu ZX, Li X, Yang ZY, Xie F. Novel structure with negative Poisson's ratio and enhanced Young's modulus. Composite Structures. 2016;138:243-52.

[18] Fu M, Chen Y, Hu L. A novel auxetic honeycomb with enhanced in-plane stiffness and buckling strength. Composite Structures. 2017;160:574-85.

[19] Li D, Yin J, Dong L, Lakes RS. Strong re-entrant cellular structures with negative Poisson's ratio. Journal of Materials Science. 2018;53:1-7.

[20] Ma F, Ying Z, Liang H, Wang J. Effects of Cell Microtopology on the In-plane Dynamic Crushing Analysis of Re-entrant Square Cellular Material. Automotive Innovation. 2018;1:24-34.

[21] Ingrole A, Hao A, Liang R. Design and modeling of auxetic and hybrid honeycomb structures for in-plane property enhancement. Materials \& Design. 2017;117:72-83.

[22] Wang H, Lu Z, Yang Z, Li X. A novel re-entrant auxetic honeycomb with enhanced in-plane impact resistance. Composite Structures. 2019;208:758-70.

[23] Mukhopadhyay T, Adhikari S, Batou A. Frequency domain homogenization for the viscoelastic properties of spatially correlated quasi-periodic lattices. International Journal of Mechanical Sciences. 2019;150:784-806.

[24] Mukhopadhyay T, Adhikari S. Effective in-plane elastic properties of auxetic honeycombs with spatial irregularity. Mechanics of Materials. 2016;95:204-22.

[25] Sun Y, Wang B, Pugno N, Wang B, Ding Q. In-plane stiffness of the anisotropic multifunctional hierarchical honeycombs. Composite Structures. 2015;131:616-24.

[26] Sun Y, Pugno NM. In plane stiffness of multifunctional hierarchical honeycombs with negative Poisson's ratio sub-structures. Composite Structures. 2013;106:681-9.

[27] Zhang Y, Lu M, Wang CH, Sun G, Li G. Out-of-plane crashworthiness of bio-inspired self-similar regular hierarchical honeycombs. Composite Structures. 2016;144:1-13.

[28] Fang J, Sun G, Qiu N, Pang T, Li S, Li Q. On hierarchical honeycombs under out-of-plane crushing. International Journal of Solids and Structures. 2018;135:1-13.

[29] Wu Y, Fang J, He Y, Li W. Crashworthiness of hierarchical circular-joint quadrangular honeycombs. Thin-Walled Structures. 2018;133:180-91.

[30] Yin H, Huang X, Scarpa F, Wen G, Chen Y, Zhang C. In-plane crashworthiness of bio-inspired hierarchical honeycombs. Composite Structures. 2018;192:516-27.

[31] Xu X, Zhang Y, Wang J, Jiang F, Wang CH. Crashworthiness design of novel hierarchical hexagonal columns. Composite Structures. 2018;194:36-48.

[32] Qiao J, Chen C. In-plane crushing of a hierarchical honeycomb. International Journal of Solids and Structures. 2016;85-86:57-66.

[33] Tang T, Zhang W, Yin H, Wang H. Crushing analysis of thin-walled beams with various section geometries under lateral impact. Thin-Walled Structures. 2016;102:43-57.

[34] Wang G, Kou LY, Cong-Chang XU, Tuo YE, Luo-Xing LI. Finite Element Modeling for Compression Fracture Behavior of6000Aluminum Alloys Thin-Walled Structure. Materials for Mechanical Engineering. 2016.

[35] Ye T, Wang G, Yao ZQ, Li LX. Ductile fracture behavior of 6xxx aluminum alloy thin-walled components of automobile. Chinese Journal of Nonferrous Metals. 2014;24:878-87.

[36] Ajdari A, Jahromi BH, Papadopoulos J, Nayeb-Hashemi H, Vaziri A. Hierarchical honeycombs with tailorable properties. International Journal of Solids \& Structures. 2012;49:1413-9.

[37] Khan MK, Baig T, Mirza S. Experimental investigation of in-plane and out-of-plane crushing of aluminum honeycomb. Materials Science and Engineering: A. 2012;539:135-42.

[38] Qiu XM, Zhang J, Yu TX. Collapse of periodic planar lattices under uniaxial compression, part I: Quasi-static strength predicted by limit analysis. International Journal of Impact Engineering. 2009;36:1223-30. 